\title{
An Axiomatic Basis for Bidirectional Programming
}

\author{
HSIANG-SHANG KO, National Institute of Informatics, Japan \\ ZHENJIANG HU*, National Institute of Informatics, Japan
}

Among the frameworks of bidirectional transformations proposed for addressing various synchronisation (consistency maintenance) problems, Foster et al's [2007] asymmetric lenses have influenced the design of a generation of bidirectional programming languages. Most of these languages are based on a declarative programming model, and only allow the programmer to describe a consistency specification with ad hoc and/or awkward control over the consistency restoration behaviour. However, synchronisation problems are diverse and require vastly different consistency restoration strategies, and to cope with the diversity, the bidirectional programmer must have the ability to fully control and reason about the consistency restoration behaviour. The putback-based approach to bidirectional programming aims to provide exactly this ability, and this paper strengthens the putback-based position by proposing the first fully fledged reasoning framework for a bidirectional language - a Hoare-style logic for Ko et al's [2016] putback-based language BIGUL. The Hoarestyle logic lets the BIGUL programmer precisely characterise the bidirectional behaviour of their programs by reasoning solely in the putback direction, thereby offering a unidirectional programming abstraction that is reasonably straightforward to work with and yet provides full control not achieved by previous approaches. The theory has been formalised and checked in AGDA, but this paper presents the Hoare-style logic in a semi-formal way to make it easily understood and usable by the working BIGUL programmer.

CCS Concepts: • Software and its engineering $\rightarrow$ Domain specific languages; $\bullet$ Theory of computation $\rightarrow$ Axiomatic semantics; Hoare logic;

Additional Key Words and Phrases: asymmetric lenses, putback-based bidirectional programming

ACM Reference Format:

Hsiang-Shang Ko and Zhenjiang Hu. 2018. An Axiomatic Basis for Bidirectional Programming. Proc. ACM Program. Lang. 2, POPL, Article 41 (January 2018), 29 pages. https://doi.org/10.1145/3158129

\section{INTRODUCTION}

The need for synchronisation - or consistency maintenance - is pervasive in computing. A simple but typical example is synchronisation among documents of different formats, in which case consistency means that the documents have the same content; whenever the content of one document is modified, the other documents should also be updated to restore the consistency. Over the past decade, frameworks of bidirectional transformations have been proposed to address a diverse range of synchronisation problems [Czarnecki et al. 2009]. One such framework is Foster et al.'s [2007] asymmetric lenses, which are highly influential such that the term bidirectional programming has become largely synonymous with lens-based approaches (including lens combinators and bidirectionalisation; see, e.g., Foster et al. [2012]). Asymmetric lenses are designed for synchronising

*Also with SOKENDAI (The Graduate University for Advanced Studies).

Authors' addresses: Hsiang-Shang Ko, National Institute of Informatics, 2-1-2 Hitotsubashi, Chiyoda-ku, Tokyo, 101-8430, Japan, hsiang-shang@nii.ac.jp; Zhenjiang Hu, National Institute of Informatics, 2-1-2 Hitotsubashi, Chiyoda-ku, Tokyo, 101-8430, Japan, hu@nii.ac.jp.

This work is licensed under a Creative Commons Attribution 4.0 International License.

(C) 2018 Copyright held by the owner/author(s).

2475-1421/2018/1-ART41

https://doi.org/10.1145/3158129

Proceedings of the ACM on Programming Languages, Vol. 2, No. POPL, Article 41. Publication date: January 2018. 
two pieces of data where one side, which is called the source, has more information than the other, which is called the view. Typically, a lens program describes a forward get transformation that computes a consistent view from a source; whenever the source is modified, get is rerun to produce a new consistent view. Conversely, from the same lens program we can derive a backward put transformation that takes a source and a (possibly modified) view, and produces an updated source that is consistent with the view and can retain some information of the original source.

By definition, the two transformations derived from any lens program should satisfy two inverselike well-behavedness laws called PUtGet and GetPut (which will be formally stated in Theorem 2.2). Stevens [2010, Section 4.4] provided a revealing perspective to understand these well-behavedness laws: the get transformation denoted by a lens can be regarded as defining a (functional and executable) consistency relation on the source and view; PUTGET then says that the put transformation will correctly restore the consistency, i.e., the updated source and the view will satisfy the consistency relation, and GETPUT says that put will perform no update if the input source and view are already consistent. From this perspective, at the root of Foster et al.'s lenses and all subsequent get-based approaches is a declarative programming model, in which the programmer specifies a consistency relation (in terms of a get transformation) and obtains a consistency restorer (a put transformation) that is guaranteed (by well-behavedness) to respect the consistency relation. Mechanisms are provided for customising the restoration behaviour, but they are usually ad hoc and/or awkward to use. This is unsatisfactory in practice, since we care not only about consistency but even more about how consistency restoration is performed; with get-based approaches it is inherently difficult to understand or control the latter aspect. (See Section 8 for further discussion.)

To be concrete, let us consider a simple synchronisation problem where the source is a pair of numbers representing the width and height of a rectangle, and the view is a single number, which is consistent with a rectangle exactly when it is equal to the width of the rectangle. With respect to this definition of consistency, there are a variety of consistency restoration strategies: given a rectangle and a view, in addition to replacing the width with the view, which is necessary for restoring the consistency,

1. we can always keep the height unchanged - this is a typical "least-change" strategy;

2. we can update the height to keep the height-to-width ratio of the rectangle - in general this can be maintaining some kind of internal consistency on the source side;

3. we can reset the height to zero if the view is different from the width - although rather drastic, this would be useful when the view side does not know how the source side maintains its internal consistency, and thus simply chooses to invalidate associated data and leave them for the source side to update later;

4. we can decide to keep or reset the height depending on whether the difference between the width and the view is small enough - this is a flexible mixture of strategies 1 and 3;

5. we can use the height as a counter that is incremented every time an inconsistency is repaired though somewhat strange, in general this can be some form of logging of source changes.

As we can see, even for a simple problem like rectangle width updating, there are already many possible update strategies; this is even more the case in complex, real-world scenarios. All the above update strategies restore the same consistency but have different retentive behaviour - the way in which the information of the original source is retained - to meet different requirements. The programmer must be empowered to fully control and reason about the retentive behaviour of their programs to be sure that it is suitable for the intended applications.

Given that there are a myriad possibilities of update strategies, what can be better than having languages for programming such strategies, capturing the myriad possibilities once and for all? Following some previous work which took exactly this putback-based programming approach [Pacheco 
et al. 2014a,b; Hu et al. 2014], Ko et al. [2016] proposed a language BIGUL (short for "Bidirectional Generic Update Language"). Like the original lenses, every BIGUL program denotes a well-behaved pair of put and get transformations; in contrast to the original lenses, BIGUL is designed to express put transformations, and lets the programmer freely specify their intended update strategies. Moreover, since put uniquely determines get by well-behavedness (Lemma 2.3), the putback-based programmer is guaranteed that the get behaviour of their put program is unambiguously specified. The putback-based approach thus offers a powerful alternative to bidirectional programming when full control is needed and the more declarative get-based approaches are not enough.

This paper strengthens the putback-based position by proposing the first fully fledged reasoning framework for a bidirectional language: building on a revised version of BIGUL, we propose a Hoare-style logic [Hoare 1969] that empowers the programmer to precisely characterise both the put and get behaviour of BIGUL programs by reasoning exclusively in the putback direction, thereby offering a unidirectional programming abstraction that is reasonably straightforward to work with and yet provides full control not achieved by get-based approaches. For example, the programmer can express strategies 1 and 3 as two BIGUL programs keepHeight and resetHeight, and with our Hoare-style logic, the programmer can prove two Hoare-style triples to make sure that the two programs correctly restore the consistency and have the intended retentive behaviour:

$$
\begin{aligned}
& \{\text { True }\} \text { keepHeight }\left\{\left(w^{\prime}, h^{\prime}\right)(-, h) v \mid w^{\prime}=v \wedge h^{\prime}=h\right\} \\
& \{\text { True }\} \text { resetHeight }\left\{\left(w^{\prime}, h^{\prime}\right)(w, h) v \mid w^{\prime}=v \wedge\left(w=v \Rightarrow h^{\prime}=h\right) \wedge\left(w \neq v \Rightarrow h^{\prime}=0\right)\right\}
\end{aligned}
$$

These two putback triples state that both keepHeight and resetHeight work on any input pairs of source and view (due to their always-true precondition) and will update the width with the view $\left(w^{\prime}=v\right)$, that keepHeight will retain the original height $\left(h^{\prime}=h\right)$, and that resetHeight will retain the height if the original width is equal to the view $\left(w=v \Rightarrow h^{\prime}=h\right)$ or reset the height otherwise $\left(w \neq v \Rightarrow h^{\prime}=0\right)$. With a bit more reasoning about output range, the programmer can also prove that the get transformations denoted by these two programs work on any input rectangle and extract its width, conforming to the consistency relation $\left(w^{\prime}=v\right)$ stated in the above triples. (The other three rectangle width updating strategies can also be dealt with in the same way.)

Here are our contributions in a nutshell: We define Hoare-style putback triples for reasoning about the put behaviour of BIGUL programs, prove that they are sound and complete, and show how they can also characterise the get behaviour to some extent (Section 3). The putback proof rules provide an axiomatic encapsulation of BIGUL's semantics, and are designed for convenient domain-specific reasoning (Section 4). Uniquely, to adequately characterise get behaviour, our Hoare-style logic also includes range triples - which are also sound and complete - for estimating the output ranges of BIGUL programs (Section 5). We further propose rules for reasoning about recursive programs (Section 6), and verify a BIGUL implementation of key-based list alignment as a showcase example (Section 7). The presentation will be preceded by a recap of asymmetric lenses (Section 2), and end with some discussion (Section 8) and conclusion (Section 9).

Everything in this paper from theorems to derivation examples has been formalised and checked in AGDA version 2.5.2 with standard library version 0.13, but the AGDA formalisation is only provided as supplementary material. This paper will focus on explaining the intuition, and present the Hoare-style logic in a semi-formal way to make it suitable for human reasoning. BIGUL is originally developed in AGDA and ported to HASKell as an embedded language, and this influences the choices of the syntax and host language used in this paper: our BIGUL syntax is a hypothetical one abstracted from the HASKELL port of BIGUL; the host functional language is total and may be thought of as AGDA imperfectly disguised as HASKeLL - we will use some standard Haskell types and functions, and allow some general recursion and partiality justifiable in a total setting. 


\section{A RECAP OF ASYMMETRIC LENSES}

We start from a brief recap of some general facts about asymmetric lenses, but state these facts directly in terms of BIGUL - think of this section and the next as an introduction to the overall framework, rather than a detailed introduction to BIGUL (which will be offered in Section 4).

Every BIGUL program denotes an asymmetric lens, which is a well-behaved pair of put and get transformations. This is made precise by Definition 2.1 and Theorem 2.2.

Definition 2.1. A BIGUL program $b$ (whose possible forms are summarised in Figure 1) operating on source type $S$ and view type $V$ is assigned the type $S \hookleftarrow V$, and has two semantics:

put $b: S \rightarrow V \rightarrow$ Maybe $S$

get $b: S \rightarrow \quad$ Maybe $V$

The put - or putback - semantics is also called the backward semantics, and the get semantics is also called the forward semantics. ${ }^{1}$

As noted by Ko et al. [2016], the two transformations in Definition 2.1 are potentially partial computations modelled explicitly as total Maybe-computations. That is, put $b$ and get $b$ may fail to compute a result, in which case they produce Nothing; otherwise they return their result wrapped within the fust constructor.

Theorem 2.2 (well-Behavedness). Any BiGUL program $b$ satisfies the following two wellbehavedness laws:

$$
\begin{array}{ll}
\forall s, v, s^{\prime} . & \text { put } b s v=\text { fust } s^{\prime} \Rightarrow \text { get } b s^{\prime}=\text { fust } v \\
\forall s, v . \quad \text { get } b s=\text { fust } v \Rightarrow \text { put } b s v=\text { fust } s
\end{array}
$$

As noted by Ko et al., Theorem 2.2 gives a stronger well-behavedness guarantee [Macedo et al. 2013; Pacheco et al. 2014a] than the original definition of Foster et al. [2007] - in the original PutGet, for example, a successful put computation does not guarantee the success of the subsequent get computation. Even so, this theorem is not as practically useful as it seems because non-wellbehavedness is merely swept under the partiality carpet: both put $b$ and get $b$ perform various checks at runtime to detect possible violations of well-behavedness, and if the programmer does not pay enough attention to well-behavedness requirements, the execution of put $b$ or get $b$ can unexpectedly fail one of these runtime checks (thereby satisfying PUtGet or GETPUT vacuously). On the other hand, the theorem is still somewhat helpful since the BiGUL programmer no longer needs to worry about well-behavedness and can concentrate on totality, i.e., making sure that BIGUL programs can compute successfully on the inputs that the programmer cares about.

Well-behavedness implies that a putback transformation uniquely determines the corresponding forward transformation.

Lemma 2.3 (Foster [2009, Lemma 2.2.5]). Let $l, r: S \hookleftarrow V$. If put $l=$ put $r$ then get $l=$ get $r$.

This lemma distinguishes asymmetric lenses from other models of bidirectional transformations (e.g., Hofmann et al.'s [2011] symmetric lenses), and is the motivation behind BIGUL's putbackbased design, as it shows that it is theoretically feasible that the BIGUL programmer can think and program solely in the putback direction and still unambiguously specify the forward behaviour. This lemma does not help to explain what putback-based thinking is, though - how does the programmer actually write a putback program while understanding its forward behaviour? The key idea of this paper is that a Hoare-style logic can help to explain how that is achieved.

\footnotetext{
${ }^{1}$ In this paper we will provide an axiomatic semantics as the only formal definition of BIGUL's semantics, and omit the definitions of put and get (except for a few simple cases in Section 4.1) and proofs that rely essentially on them (like the proof of Theorem 2.2). All the definitions and proofs are included in the supplementary AGDA formalisation for reference.
} 


\section{THEORY OF PUTBACK TRIPLES}

Programming a putback transformation in BIGUL is comparable to programming with states to some extent: the BIGUL programmer is given a source state and a view state, and manipulates the two states with the aim of transferring all information in the view to the source; in the end, the updated source state is returned as the result. Reasoning about BIGUL programs thus consists of tracking the properties satisfied by the states at each step, and it follows that a Hoare-style logic is well suited for performing this kind of reasoning about states. We will introduce a set of Hoare-style triples for saying when BIGUL programs (as putback transformations) can compute successfully and return results satisfying some specified properties. Before doing so, we first need to fix our notation of relations (for specifying preconditions and postconditions).

Notation 3.1. Relations on types $A_{1}, A_{2}, \ldots, A_{n}$ are assigned the type $\mathcal{P}\left(A_{1} \times A_{2} \times \cdots \times A_{n}\right)$. When a relation $R$ of this type relates $a_{1}: A_{1}, a_{2}: A_{2}, \ldots, a_{n}: A_{n}$, we write $R a_{1} a_{2} \ldots a_{n}$.

Definition 3.2. A putback triple is a BIGUL program $b: S \hookleftarrow V$ surrounded by two putback assertions:

\section{$\{R\} \quad b \quad\left\{R^{\prime}\right\}$}

where $R: \mathcal{P}(S \times V)$ is the precondition (on the original source and the view) and $R^{\prime}: \mathcal{P}(S \times S \times V)$ is the postcondition (on the updated source, the original source, and the view). ${ }^{2}$ Valid putback triples are inductively defined by the proof rules in Figure 2 (which will be explained in Section 4).

The intended meaning of a putback triple $\{R\} b\left\{R^{\prime}\right\}$ is more or less standard: if the original source and the view satisfy the precondition $R$, then put $b$ will successfully produce an updated source satisfying the postcondition $R^{\prime}$, which can relate the updated source to the original source and the view. We have proved that putback triples are sound and complete with respect to BIGUL's put semantics.

THEOREM 3.3 (SOUNDNESS AND COMPLETENESS OF PUTBACK TRIPLES). Let $b: S \hookleftarrow V, R: \mathcal{P}(S \times V)$, and $R^{\prime}: \mathcal{P}(S \times S \times V)$.

$$
\{R\} b\left\{R^{\prime}\right\} \quad \text { if and only if } \forall s, v . R s v \Rightarrow \exists s^{\prime} \text {. put } b s v=\text { fust } s^{\prime} \wedge R^{\prime} s^{\prime} s v \text {. }
$$

Given that putback behaviour completely determines forward behaviour (Lemma 2.3), and that putback triples are about putback behaviour, shouldn't putback triples tell us something about forward behaviour as well? This is indeed the case, as will be shown by Theorem 3.8. Its statement will make use of some important definitions and notational conventions that will also be used throughout this paper.

Definition 3.4. A comprehension relation of type $\mathcal{P}\left(A_{1} \times A_{2} \times \cdots \times A_{n}\right)$ has the form

$$
\left.\left\langle\text { pat }_{1} \text { pat }_{2} \ldots \text { pat }_{n}\right| \text { prop }\right\rangle
$$

where each $p t_{i}$ is a pattern for elements of type $A_{i}$ and prop is a proposition that can refer to the variables in the patterns. The patterns we use in the paper include variables, constructors, and the wildcard pattern '_'. The relation holds for $a_{1}: A_{1}, a_{2}: A_{2}, \ldots, a_{n}: A_{n}$ exactly when each $a_{i}$ matches pat $_{i}$ and prop holds after substituting the matched components for the corresponding pattern variables.

Notation 3.5. We usually omit the proposition part of a comprehension relation when the proposition is trivially true, keeping only the pattern part. For example, $\left\langle\left({ }_{-}::_{-}\right)\right\rangle$holds exactly for non-empty lists, and $\left\langle_{-}\right\rangle$is the always-true binary relation.

\footnotetext{
${ }^{2}$ We do not require preconditions and postconditions to be syntactic entities drawn from a particular logic, but instead treat them semantically and will freely use whatever relations that are mathematically expressible.
} 


$$
\begin{aligned}
& \overline{\text { fail }: S \hookleftarrow V} \frac{f: S \rightarrow V}{\text { replace }: S \hookleftarrow S} \frac{l: S \hookleftarrow V \quad r: T \hookleftarrow W}{\operatorname{skip~} f: S \hookleftarrow V} \quad \frac{l * r:(S \times T) \hookleftarrow(V \times W)}{l *(V)}
\end{aligned}
$$

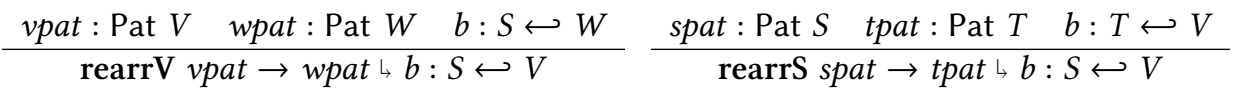

$$
\begin{aligned}
& \frac{b s:(\text { Branch } S V)^{*}}{\text { case }\llcorner b s: S \hookleftarrow V} \quad \frac{M: \mathcal{P}(S \times V) \quad E: \mathcal{P} S \quad b: S \hookleftarrow V}{\text { normal } M \text { exit } E\llcorner b: \text { Branch } S V} \quad \frac{M: \mathcal{P}(S \times V) \quad f: S \rightarrow V \rightarrow S}{\text { adaptive } M\llcorner f: \text { Branch } S V}
\end{aligned}
$$

Fig. 1. BIGUL constructs and their typing (simplified). Pat and $(-)^{*}$ are hypothetical type constructors for patterns and sequences respectively. The symbol ' $L$ ' indicates that its right-hand side is syntactically a sub-node of its left-hand side; in displayed code, the right-hand side is typeset in an indented block.

Notation 3.6. The angle brackets delimiting a comprehension relation may be omitted where delimitation is unnecessary, like in an assertion containing only a comprehension relation. For example, $\{\langle s v \mid s=v\rangle\}$ is abbreviated to $\{s v \mid s=v\}$.

Definition 3.7. The graph of a function $f: A \rightarrow$ Maybe $B$ is a relation $\mathcal{G} f: \mathcal{P}(A \times B)$ which relates $a: A$ and $b: B$ exactly when $f a=$ fust $b$.

Theorem 3.8 (partial Forward Consistency). Let $b: S \hookleftarrow V, R: \mathcal{P}(S \times V)$, and $C: \mathcal{P}(S \times V)$.

$$
\text { If }\{R\} b\left\{s^{\prime}-v \mid C s^{\prime} v\right\} \text { then } \mathcal{G}(\text { get } b) \cap R \subseteq C \text {. }
$$

Proof. Suppose get $b s=$ fust $v$ and $R s v$. The latter assumption triggers Theorem 3.3, so we know that put $b s v=$ fust $s^{\prime}$ for some $s^{\prime}$ and that $C s^{\prime} v$ holds. On the other hand, by GetPut, we can turn the first assumption get $b s=$ fust $v$ into put $b s v=$ fust $s$. Seeing that put $b s v$ computes to both $s^{\prime}$ and $s$, we can deduce $s^{\prime}=s$, and thus having $C s^{\prime} v$ is the same as having $C s v$.

That is, if we can prove that a putback transformation establishes consistency $C$ between the updated source and the view, then, roughly speaking, a part of the behaviour of the corresponding forward transformation will be constrained by $C$. We call Theorem 3.8 partial forward consistency for two reasons. The first reason is that Theorem 3.8 does not guarantee that the entire graph of the forward transformation will be contained in $C$ - the containment is guaranteed only for the part of the graph that falls within $R$. In practice, this makes Theorem 3.8 not very helpful unless $R$ is always true, in which case the entire graph will indeed be contained in $C$. Even in this case, though, there is still the second reason: Theorem 3.8 says nothing about the totality of the forward transformation, i.e., on which subset of sources the forward transformation can successfully produce results. We will augment Theorem 3.8 to get a practically useful version (Theorem 5.4). But before that, let us look at the concrete putback proof rules and some examples of putback reasoning.

\section{BIGUL AND THE PUTBACK PROOF RULES}

In this section we introduce BIGUL's constructs (Figure 1) and their putback proof rules (Figure 2). For each construct, we will give its type - which is essential for inferring the types of entities in assertions - and explain the corresponding proof rule with the help of an operational intuition about the construct.

Note that assertions are intended to be semantic rather than syntactic - for example, if the precondition stated in a rule is $\left\langle_{-}\right\rangle$, we will regard the rule as directly applicable when the actual precondition is, say, $\langle s v \mid s=s \wedge v=v\rangle$, which differs from $\left\langle_{-}\right\rangle$syntactically but still denotes the always-true binary relation semantically. 


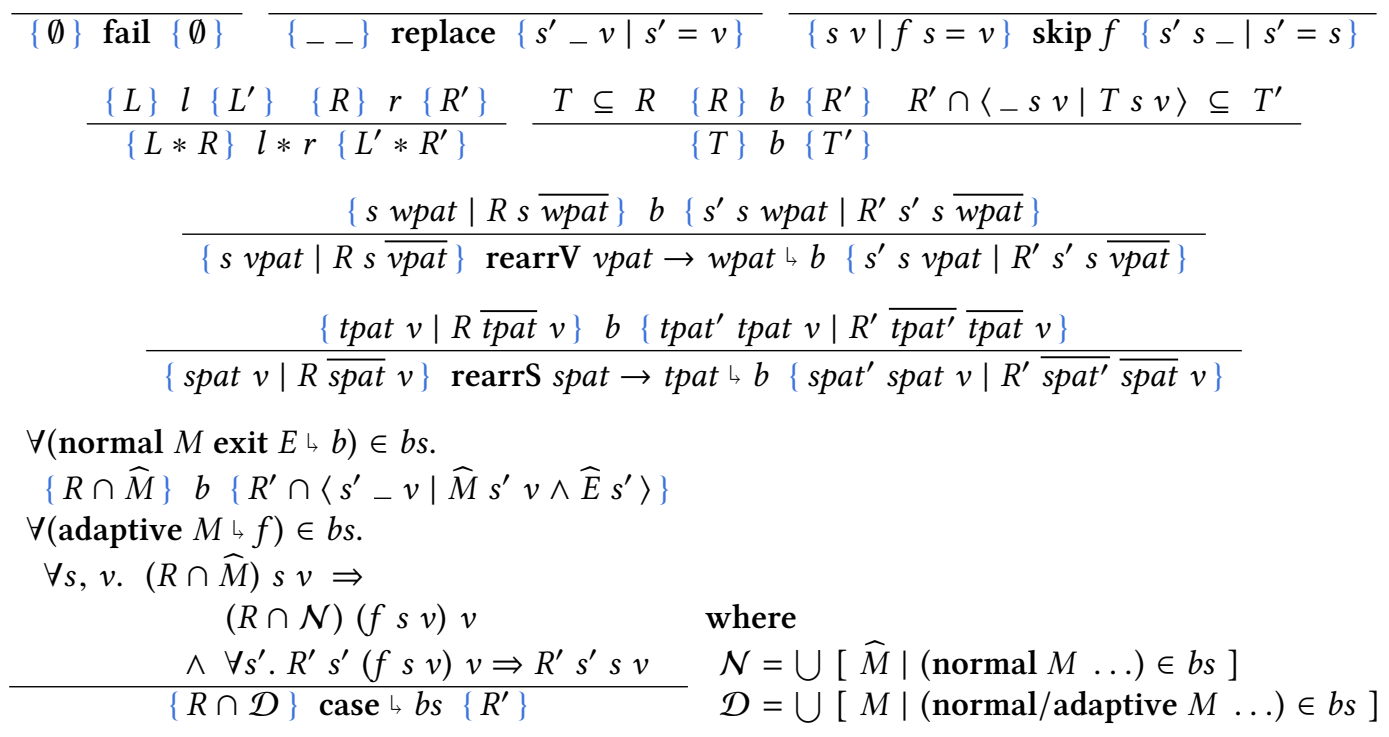

Fig. 2. Putback proof rules. $\widehat{M}$ denotes the "actual main condition" of a branch: the main condition $M$ of the branch intersected with the negations of the main conditions of all the previous branches. "Actual exit conditions" $\widehat{E}$ are analogous.

\subsection{Atomic Constructs}

BIGUL has three atomic constructs, whose corresponding rules are in the first row of Figure 2.

The fail construct has type $S \hookleftarrow V$ for any types $S$ and $V$. The precondition of the fail rule is the empty relation $\emptyset$, saying that no input can make fail compute successfully. This directly corresponds to the implementation: put fail $s v=$ Nothing.

The replace construct has type $S \hookleftarrow S$ for any type $S$, and replaces the source with the view regardless of what they are, i.e., put replace $s v=$ fust $v$. Correspondingly, the precondition of the replace rule is the always-true relation, and the postcondition states that the updated source $s^{\prime}$ will be equal to the view $v$.

The skip construct takes a function $f: S \rightarrow V$ in the host language as an argument and has type $S \hookleftarrow V$. It ignores the view and leaves the source as it is; correspondingly, the postcondition says that the updated source $s^{\prime}$ will be equal to the original source $s$. Unlike replace, we cannot skip under all circumstances - before throwing the view away, we must ensure that it can be recovered from the source, or otherwise there is no hope to establish PUTGET. The precondition thus requires that the view can be computed from the source by $f$. In the implementation, this precondition is checked dynamically: put (skip $f) s v=$ if $f s==v$ then fust $s$ else Nothing.

\subsection{Product}

Given two BIGUL programs $l: S \hookleftarrow V$ and $r: T \hookleftarrow W$, we can form the product of the two programs $l * r:(S \times T) \hookleftarrow(V \times W)$, with $l$ operating on the first components and $r$ on the second components. If two putback triples with preconditions $L$ and $R$ have been established for $l$ and $r$, the precondition of the product program will be

$$
L * R=\langle(s, t)(v, w) \mid L s v \wedge R t w\rangle
$$


The relation-level ' $*$ ' operator can be seen as a simple variant of separating conjunction [Reynolds 2002], and can be defined arity-generically to construct a relation on $n$ pairs from an $n$-ary relation on all the first components and the other on all the second components. The postcondition can then be stated also in terms of this ' $*$ ' operator.

Example 4.1 (parallel replacement). We can now construct simple derivations like the following one for replace $*$ replace, which we use as an example to explain our derivation format:

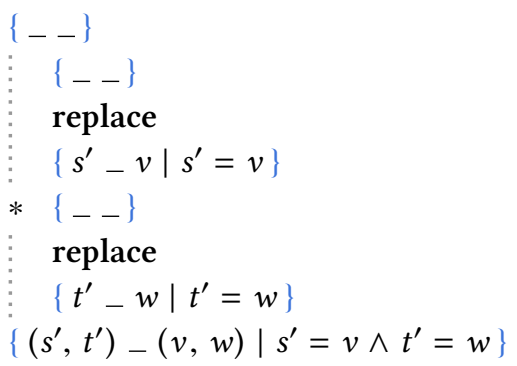

First note that the syntax tree structure of replace $*$ replace is reflected in indentation: the top-level node is ' $*$ ', whose two sub-nodes - both being replace - are indented to the next level. Then, following the indentation structure, the assertions are added: the assertions about a node are put on the same indentation level as the node, with the precondition and postcondition appearing respectively before and after the node. This format is compact and yet retains the tree structure of the derivation, making it easier to check the correctness of the derivation.

\subsection{The Consequence Rule}

The consequence rule we present in Figure 2 (the right one in the second row) may seem unusual, but first observe that it is a stronger version of the usual one (so at least there is nothing to lose):

$$
\frac{T \subseteq R \quad\{R\} \quad b \quad\left\{R^{\prime}\right\} \quad R^{\prime} \subseteq T^{\prime}}{\{T\}}
$$

To see why we need a stronger consequence rule, consider deriving this triple:

$$
\{-(v, w) \mid v=w\} \text { replace } * \text { replace }\left\{\left(s^{\prime}, t^{\prime}\right)_{-} \mid s^{\prime}=t^{\prime}\right\}
$$

where there is some entanglement between the first and second components in the precondition and postcondition, so the product rule is not directly applicable. We could try to extend the derivation in Example 4.1 using the usual consequence rule (1):

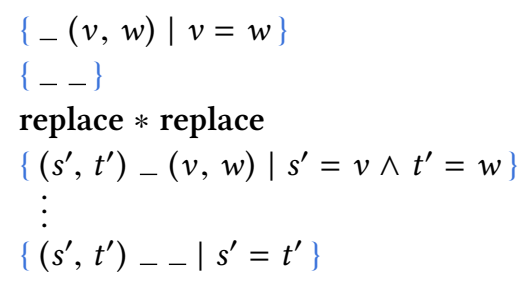

Adjacent assertions on the same indentation level indicate an invocation of the consequence rule, with the one above implying the one below. In the first two lines of this derivation, there is one such invocation, which turns $\left\langle_{-}(v, w) \mid v=w\right\rangle$ into $\left\langle_{-}\right\rangle$so that the product rule can apply. On the other hand, the postcondition that we can establish, i.e., $\left\langle\left(s^{\prime}, t^{\prime}\right)_{-}(v, w) \mid s^{\prime}=v \wedge t^{\prime}=w\right\rangle$, does not imply the postcondition we want to establish, i.e., $\left\langle\left(s^{\prime}, t^{\prime}\right){ }_{-} \mid s^{\prime}=t^{\prime}\right\rangle$. The usual consequence rule (1) can help us to get rid of the entanglement $v=w$, but that entanglement 
is needed to establish the final implication (by $s^{\prime}=v=w=t^{\prime}$ ). We thus need the stronger consequence rule to be able to carry over whatever we know about the original source and the view from the precondition to the postcondition. When working in our derivation format, the stronger consequence rule allows us to prove an implication between adjacent postconditions using whichever preconditions for the same node (on the same indentation level) as additional premises about the original source and the view. ${ }^{3}$

\subsection{Rearrangement}

A guiding intuition for BIGUL programming is to manipulate the source and view to make their shapes match, which is achieved mainly with the rearrangement operations. To provide a more concrete motivation: We have seen that the product combinator (Section 4.2) allows us to synchronise source and view tuples of arbitrary size, provided that their structures are the same. When this is not the case, in BIGUL we can use a simple class of pattern-matching $\lambda$-expressions to rearrange the source and/or the view to make them match structurally and ready for further synchronisation. For example, the height-keeping strategy 1 we proposed for the rectangle width updating problem (Section 1) can be expressed in BIGUL as:

$$
\begin{aligned}
& \text { keepHeight }:(\mathbb{N} \times \mathbb{N}) \hookleftarrow \mathbb{N} \\
& \text { keepHeight }=\text { rearrV } v \rightarrow(v,()) \\
& \text { replace -- const is the } \mathrm{K} \text { combinator (const } x y=x \text { ), and } \\
& \text { * skip const () -- '()' is the sole inhabitant of the unit type }
\end{aligned}
$$

Initially, the view is a single number, whereas the source is a pair. To make their structures match, we use the view rearrangement operation rearrV $v \rightarrow(v,())$ to apply the $\lambda$-expression $\lambda v \rightarrow(v,())$ to the view and make the result the new view. Inside the rearrV, the source and view are both pairs, so we can use replace $*$ skip const () to update the width and keep the height as it is. Below we will mainly discuss view rearrangement; source rearrangement is largely analogous, and will be discussed towards the end of this subsection.

View rearrangement. The general form of a view rearrangement is rearrV vpat $\rightarrow$ wpat $\iota b$ : $S \hookleftarrow V$, where vpat is a pattern for the original view type $V$, wpat is a "pattern" for a new view type $W$, and the inner program $b$ has type $S \hookleftarrow W$. (The symbol ' $\iota$ ' indicates that $b$ is syntactically a sub-node of 'rearrV vpat $\rightarrow$ wpat'; in displayed code, $b$ is typeset in an indented block below 'rearrV vpat $\rightarrow$ wpat'.) The intention is to represent a closed $\lambda$-expression $\lambda$ vpat $\rightarrow$ wpat to be applied to the view. Strictly speaking, wpat is not a pattern but an expression, which can be built using variables in vpat and constructors. (Apart from the fact that wpat looks similar to a pattern, we will explain why it is beneficial to think of wpat as a pattern shortly.) Wildcards are not allowed in vpat, and all variables in vpat must appear in wpat and can appear multiple times these synctactic restrictions ensure that the $\lambda$-expression is invertible, or, more intuitively speaking, does not lose information.

The view rearrangement rule. Intuitively, a rearrangement only massages the state into an alternate shape suitable for further processing, rather than applying an arbitrary and distorting transformation. This intuition significantly influences the design of the rearrangement rules, which reflect that rearrangement is essentially just a "change of perspective". If we are rearranging the view

\footnotetext{
${ }^{3}$ Alternatively and equivalently, as suggested by an anonymous reviewer of an earlier version of this paper, we can keep the usual consequence rule (1) and introduce a separation logic-style frame rule: $\{R\} \quad b \quad\left\{R^{\prime}\right\} \Rightarrow$ $\{R \cap T\} b\left\{R^{\prime} \cap\langle-s v \mid T s v\rangle\right\}$, which is somewhat more elegant. However, we feel that being able to use preconditions to establish implications between postconditions is more convenient in practice, and the stronger consequence rule captures this ability more directly.
} 
from vpat to wpat, it must mean that the view matches vpat right before the rearrangement, and we should be able to state properties satisfied by the view at that point in terms of its components. The precondition for rearrV is thus a comprehension relation:

$$
\langle s v p a t \mid R s \overline{v p a t}\rangle
$$

which requires that the view matches vpat and that any properties about the view are stated in terms of the variables in vpat, which we denote by $\overline{v p a t}$. After the rearrangement, due to the invertibility restrictions, the new view will retain all the components of the original view; the components may be shuffled around and duplicated, but whatever we knew about the components will remain true. The precondition for the inner program $b$ is thus:

$\langle s w p a t \mid R s \overline{w p a t}\rangle$

This inner precondition asserts that the new view matches wpat and that whatever holds for $\overline{v p a t}$ in the outer precondition also holds here for $\overline{w p a t}$, which is the same as $\overline{v p a t}$ because of the invertibility restrictions. Being able to state this inner precondition is the reason that we think of wpat also as a pattern, even though that means in general we have to allow non-linear patterns, where multiple occurrences of the same variable indicate implicitly that values at those positions should be equal. When using the rule in actual derivations, the two preconditions will differ only in their view patterns and have the same proposition part. Therefore, the view rearrangement rule only changes the shape we expect the view to take, not the properties we know about the content of the view. This change-of-perspective interpretation works for the postconditions as well.

Example 4.2 (rectangle width updating - keeping the height). We can now verify keepHeight as follows, where the precondition (assertion 1) is always true and the postcondition (assertion 2) says that the consistency will be established $\left(w^{\prime}=v\right)$ and the height will be retained $\left(h^{\prime}=h\right)$ :

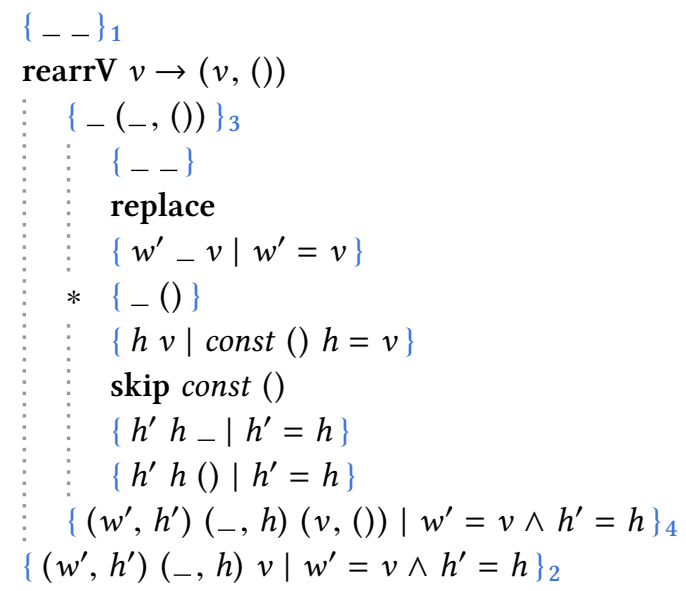

Note that when constructing the derivation inwards from the initial precondition and postcondition, it is effortless to push them inside the rearrV and turn them into assertions 3 and 4 just by changing the view pattern to a pair pattern, as instructed by the rearrV.

Source rearrangement and the corresponding rule. Analogous to view rearrangement, the general form of a source rearrangement is rearrS spat $\rightarrow$ tpat $\iota b: S \hookleftarrow V$, where spat is a pattern for the original source type $S$, tpat is a pattern for the new source type $T$, and the inner program $b$ has type $T \hookleftarrow V$. The same syntactic restrictions for invertibility apply to spat and tpat. Operationally, the source is transformed using $\lambda$ spat $\rightarrow$ tpat, and $b$ is executed on the new source and the view; after that, the updated source must match tpat, and will be transformed back to the shape of 
spat (as if evaluating $\lambda$ spat $\rightarrow$ tpat backwards). Dual to the view rearrangement rule, the source rearrangement rule also reflects a change of perspective by varying the source patterns. Notably, the postcondition for $b$

$$
\left\langle\text { tpat }{ }^{\prime} \text { tpat } v \mid R^{\prime} \overline{\text { tpat' }} \overline{\text { tpat }} v\right\rangle
$$

says explicitly that the updated source produced by $b$ should match tpat' (which is just tpat with its variables freshly renamed, to avoid name clashes with those variables in the other occurrence of tpat), which is a requirement often overlooked by novice BIGUL programmers.

Example 4.3 (view equality checking). The following small program implements the equality checking operator in reversible programming (see, e.g., Thomsen and Axelsen [2015]):

$$
\begin{array}{rl}
\text { eqCheck }: E q A & A A \hookleftarrow(A \times A) \\
\text { eqCheck }= & \text { rearrS } x \rightarrow(x, x) \\
\vdots \quad \text { replace }
\end{array}
$$

where the ' $E q A$ ' constraint in the type indicates that we need decidable equality on $A$ for the program to be executable. This example shows that rearrS can impose restrictions on the result produced by the inner program: Operationally, the source is rearranged with the $\lambda$-expression $\lambda x \rightarrow(x, x)$, and then the inner program replace is executed, after which the rearranging $\lambda$ expression is evaluated backwards by matching the replaced source with the non-linear pattern $(x, x)$ - in effect checking whether the components are equal - and then returning one of the components. For this computation to succeed, the replaced source - i.e., the input view - must be a pair of duplicate values. In the derivation for eqCheck below, this restriction appears in assertion 1 (as the non-linear pattern $\left(s^{\prime}, s^{\prime}\right)$ for the updated source), arising from the use of the rearrS rule.

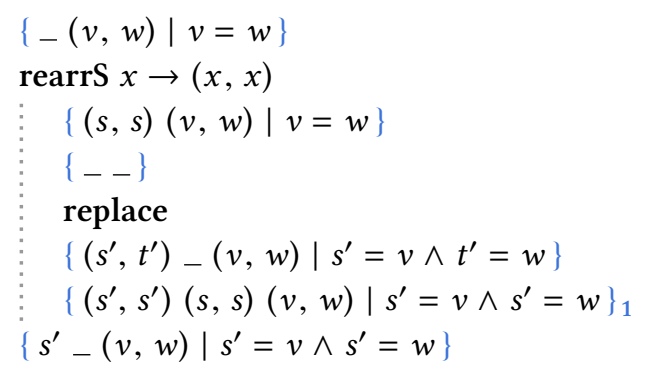

\subsection{Case Analysis}

More sophisticated programs require case analysis, for which BIGUL provides a powerful and intricate case construct. For a simple example, the height-resetting strategy 3 for the rectangle width updating problem (Section 1) can be expressed as:

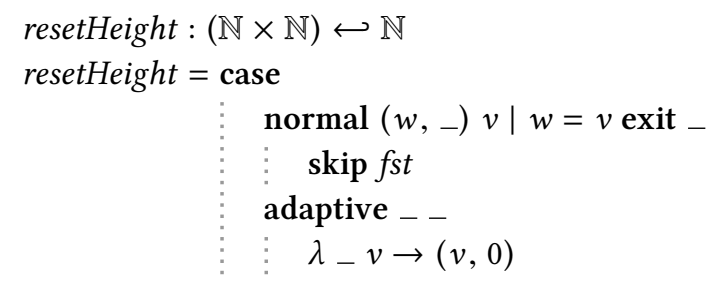

Roughly speaking, this program checks whether the width of the source is equal to the view, and skips if that is the case; otherwise, it creates a new rectangle whose width is the view and whose height is zero. 
Syntax of case. In general, a case analysis in BIGUL has the form case $\downarrow$ bs $: S \hookleftarrow V$ where $b s$ is a sequence of normal or adaptive branches. For normal branches, the general form is normal $M$ exit $E\llcorner b$ where $M: \mathcal{P}(S \times V)$ is called the main condition, $E: \mathcal{P} S$ is called the exit condition, and $b: S \hookleftarrow V$ is the branch body. For adaptive branches, the general form is adaptive $M \hookrightarrow f$ where $M: \mathcal{P}(S \times V)$ is again the main condition, and $f: S \rightarrow V \rightarrow S$ is a function in the host language. The syntactic conventions described by Notation 3.5 and Notation 3.6 are also adopted for comprehension relations used as main or exit conditions. ${ }^{4}$

The case rule. Operationally, the execution of a case finds the first branch whose main condition is satisfied by the source and view, and enters that branch. Suppose that the precondition and postcondition we want to verify for the entire case are $R$ and $R^{\prime}$ respectively. The case rule in Figure 2 says that the precondition should be restricted to $R \cap \mathcal{D}$ where $\mathcal{D}$ is the union of all the main conditions, so that the precondition is strong enough to guarantee that some branch will be entered. The rest of the job is to verify each branch.

Normal branches. If a normal branch normal $M$ exit $E\llcorner b$ is entered, its body $b$ is executed; the case rule thus requires us to verify the behaviour of $b$ by deriving the following triple:

$$
\{R \cap \widehat{M}\} \quad b \quad\left\{R^{\prime} \cap\left\langle s^{\prime}-v \mid \widehat{M} s^{\prime} v \wedge \widehat{E} s^{\prime}\right\rangle\right\}
$$

The precondition is strengthened with the main condition since we know that the source and view must satisfy the main condition if the branch is entered. However, the precise condition satisfied is not $M-$ since the branches are tried in order and a branch is entered only when the main conditions of all the previous branches are not satisfied, we should regard the actual main condition of a branch as $M$ intersected with the negations of the main conditions of all the previous branches. We denote this actual main condition by $\widehat{M}$, and the precondition for $b$ is strengthened to $R \cap \widehat{M}$. As for the postcondition, in addition to $R^{\prime}$, we also require (i) that the updated source and the view satisfy the actual main condition $\widehat{M}$ and (ii) that the updated source satisfy the actual exit condition $\widehat{E}$, which is $E$ intersected with the negations of the exit conditions of all the previous normal branches. These requirements are essential for guaranteeing well-behavedness; for a detailed development of these requirements, see $\mathrm{Hu}$ and $\mathrm{Ko}$ [2017, Section 5.5].

Adaptive branches. Requirement (i) above for normal branches turns out to be very restrictive, making normal branches only capable of dealing with "almost consistent" cases in practice. However, we often need branches whose main condition describes a particular kind of inconsistency and whose purpose is to repair that inconsistency - that is, their main conditions are supposed to be broken after updating, and this is against the nature of normal branches. Instead, for repairing inconsistency, we use adaptive branches, which are comparable with Foster et al.'s [2007] "fixup functions". When entered, an adaptive branch adaptive $M\llcorner f$ applies $f$ to the source and view to produce an adapted source; this adapted source then takes the place of the original source, and the whole case is rerun. Naturally, requirements have to be imposed on $f$, as stated in the case rule:

$$
\begin{aligned}
\forall s, v . \quad(R \cap \widehat{M}) s v & \Rightarrow(R \cap \mathcal{N})(f s v) v \\
& \wedge \forall s^{\prime} . R^{\prime} s^{\prime}(f s v) v \Rightarrow R^{\prime} s^{\prime} s v
\end{aligned}
$$

Like normal branches, we know that $R$ and $\widehat{M}$ hold for the original source $s$ and the view $v$, and that has to be strong enough to make $s$ and $v$ satisfy two requirements:

\footnotetext{
${ }^{4}$ We make $M$ and $E$ comprehension relations to simplify the presentation - in real, executable programs, $M$ and $E$ should be "comprehension expressions" that compute to boolean values instead of propositions, but that would mess up the assertions where we would have to write propositions like $M s v=$ true instead of just $M s v$.
} 
- First, the adapted source $f s v$ and the view $v$ can make the whole case rerun successfully. That is, they should satisfy $R$, the precondition for the entire case, and also $\mathcal{N}$, which denotes the union of the actual main conditions of the normal branches - this ensures that the rerunning will go into a normal branch and terminate there, instead of revisiting adaptive branches indefinitely.

- Second, the rerunning of the case establishes the postcondition for the updated source, the adapted source, and the view, but ultimately we want the postcondition established not for the adapted source but the original source. Therefore, whichever updated source $s^{\prime}$ is produced by the rerunning, the postcondition $R^{\prime} s^{\prime}(f s v) v$ established by the rerunning has to be sufficient for the ultimate postcondition $R^{\prime} s^{\prime} s v$.

In practice, the first requirement leads us to write adaptive behaviour that performs enough inconsistency-repairing so as to be able to go back into normal branches, while the second requirement discourages us from radically changing the source during adaptation so that it is possible to derive properties about the original source from properties about the adapted source. (We will see how these two guidelines are applied in a more illustrative scenario in Section 7.)

Representing the case rule in our derivation format. Before we see some derivation examples, we need to think about how the case rule - in particular, the two requirements for adaptive branches - are to be incorporated into our derivation format. Observe that the two requirements can be rewritten as relational inclusions:

$$
\begin{aligned}
& \forall s, v .(R \cap \widehat{M}) s v \Rightarrow(R \cap \mathcal{N})(f s v) v \\
\equiv & R \cap \widehat{M} \subseteq\langle s v \mid(R \cap \mathcal{N})(f s v) v\rangle \\
& \forall s, v .(R \cap \widehat{M}) s v \Rightarrow \forall s^{\prime} . R^{\prime} s^{\prime}(f s v) v \Rightarrow R^{\prime} s^{\prime} s v \\
\equiv & \left\langle s^{\prime} s v \mid R^{\prime} s^{\prime}(f s v) v\right\rangle \cap\left\langle{ }_{-} v \mid(R \cap \widehat{M}) s v\right\rangle \subseteq R^{\prime}
\end{aligned}
$$

which match the forms of the two inclusions in the consequence rule. Indeed, if $f$ were a BIGUL operation (symbolising the rerunning of the case) such that $\{\mathcal{F}\} f\left\{\mathcal{F}^{\prime}\right\}$, where the precondition

$$
\mathcal{F}=\langle s v \mid(R \cap \mathcal{N})(f s v) v\rangle
$$

states that (before the rerunning) the adapted source $f s v$ and the view $v$ should be guaranteed to satisfy $R \cap \mathcal{N}$, and the postcondition

$$
\mathcal{F}^{\prime}=\left\langle s^{\prime} s v \mid R^{\prime} s^{\prime}(f s v) v\right\rangle
$$

states that (after the rerunning) $R^{\prime}$ is established for the updated source $s^{\prime}$, the adapted source $f s v$, and the view $v$, then we could invoke the consequence rule:

$$
\frac{R \cap \widehat{M} \subseteq \mathcal{F} \quad\{\mathcal{F}\} f\left\{\mathcal{F}^{\prime}\right\} \quad \mathcal{F}^{\prime} \cap\left\langle{ }_{-} s v \mid(R \cap \widehat{M}) s v\right\rangle \subseteq R^{\prime}}{\{R \cap \widehat{M}\} f\left\{R^{\prime}\right\}}
$$

We therefore use the following derivation format for adaptive branches:

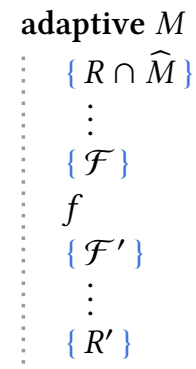


The way to think about this format is that eventually we want to establish $\{R \cap \widehat{M}\} f\left\{R^{\prime}\right\}$, but the actual precondition and postcondition of $f$ are respectively $\mathcal{F}$ and $\mathcal{F}^{\prime}$ instead, and hence we should invoke the consequence rule (2) and prove the two inclusions. Let us emphasise that assertions in adaptive branches do not really constitute triples, but are merely an organisation of the proof obligations for adaptive branches such that we can work with the proof obligations in the same way as we work with real triples.

Example 4.4 (rectangle width updating - resetting the height). Now we can verify the resetHeight program as follows, where the precondition is always true and the postcondition says that the consistency will be established and the height will be retained or reset depending on whether the view is consistent or not:

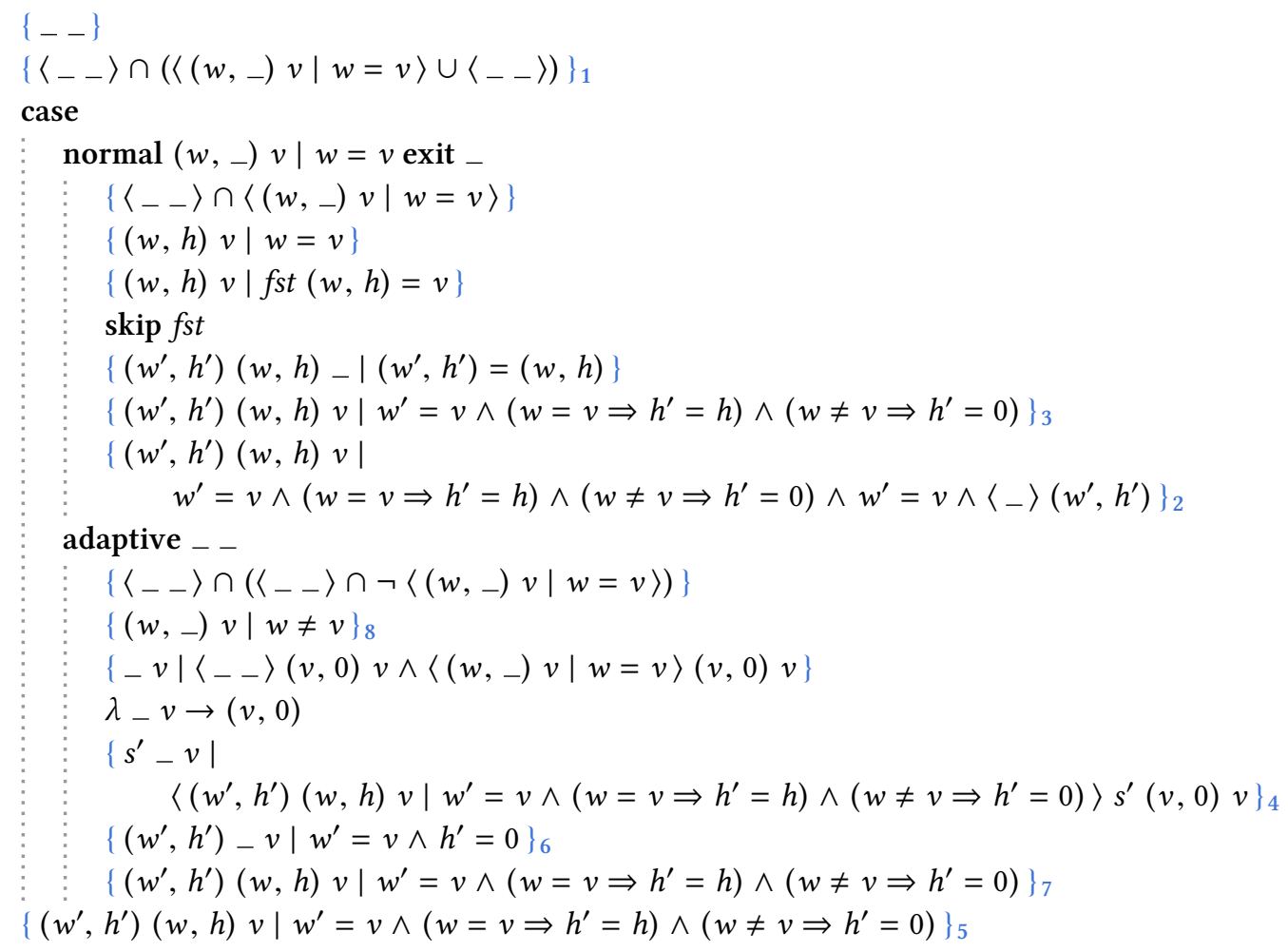

In this derivation, some assertions (like assertion 1) are given so that it is easier to compare the derivation with the generic case rule, but in practice we can often skip these assertions and see that the case rule is indeed applicable. For example, we tend to omit assertion 1 in practice since we can see that the adaptive branch is a catch-all branch; we even tend to omit assertion 2 since we can just check whether assertion 3 covers the extra conditions about the updated source, namely $w^{\prime}=v \wedge\left\langle_{-}\right\rangle\left(w^{\prime}, h^{\prime}\right)$.

What happens in the adaptive branch is worth tracing. After the rerunning of the case produces an updated source $s^{\prime}$, assertion 4 states that the postcondition (assertion 5) holds for $s^{\prime}$, the adapted source $(v, 0)$, and the view $v$. We can then deduce that the updated width $w^{\prime}$ is $v$ and, by substituting $(v, 0)$ for $(w, h)$ in the "retentive conjunct" $w=v \Rightarrow h^{\prime}=h$ in assertion 4 , that the updated height $h^{\prime}$ is zero, arriving at assertion 6. Having $h^{\prime}=0$ is sufficient for establishing the "resetting conjunct" $w \neq v \Rightarrow h^{\prime}=0$ in assertion 7, whose "retentive conjunct" $w=v \Rightarrow h^{\prime}=h$ is vacuous due to assertion 8 . 
Example 4.5 (embedding pairs of transformations into BIGUL). The resetHeight program in fact exhibits a general programming pattern: a case with a normal branch accepting consistent states and leaving the source as it is, and an adaptive branch recovering from inconsistency. We can abstract this pattern to the following emb program, which takes a pair of (total) forward and backward transformations and embeds them into BIGUL:

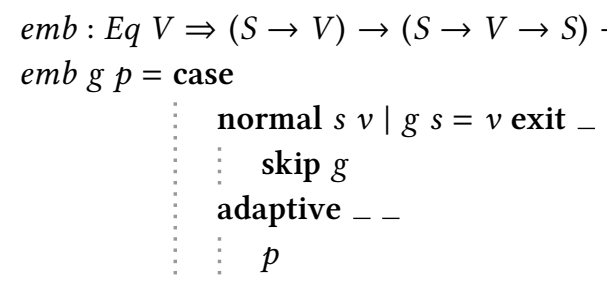

It is easy to see that resetHeight $=e m b f_{s t}\left(\lambda_{-} v \rightarrow(v, 0)\right)$. What we proved for resetHeight in Example 4.4 can be generalised to the following triple for $\mathrm{emb} g \mathrm{p}$, where $\mathrm{g}$ is used to define consistency:

$$
\left\{{ }_{-}\right\} \operatorname{emb} g p\left\{s^{\prime} s v \mid g s^{\prime}=v \wedge\left(g s=v \Rightarrow s^{\prime}=s\right) \wedge\left(g s \neq v \Rightarrow s^{\prime}=p s v\right)\right\}
$$

Interestingly, to prove this triple, we only require $g$ and $p$ to satisfy PutGet $(\forall s, v \cdot g(p s v)=v$, which is Theorem 2.2's PUtGet specialised for total functions); GetPUt of emb g p arises from the logic of emb itself and does not depend on $g$ and $p$. Indeed, in the case of resetHeight, the pair of transformations being embedded satisfies only PUTGET.

On the other hand, if we have both PutGet and GetPut $(\forall s . p s(g s)=s)$, then we can derive a stronger triple saying that the putback behaviour of emb g $p$ completely coincides with $p$ :

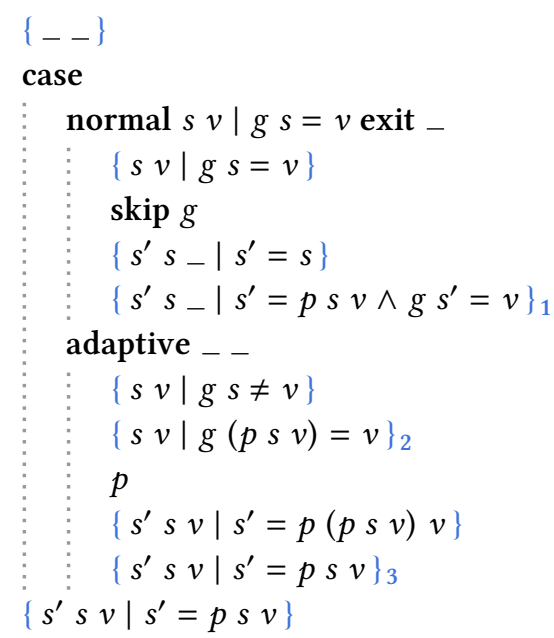

GetPut is used for assertion 1, and PutGet is used for assertion 2. Assertion 3 requires the PutTwice property $\forall s, v \cdot p(p s v) v=p s v$, which is known to follow from PutGet and GetPut (see, e.g., Fischer et al. [2015a, Section 3]).

\section{RANGE TRIPLES AND TOTAL FORWARD CONSISTENCY}

We have seen in Example 4.5 that any pair of transformations satisfying only PUTGET can be embedded into BIGUL. For example, resetHeight in Example 4.4 can be seen as emb fst reset where reset $=\lambda-v \rightarrow(v, 0)$. An alternative way to embed reset is to express it directly in terms of BIGUL's constructs: 


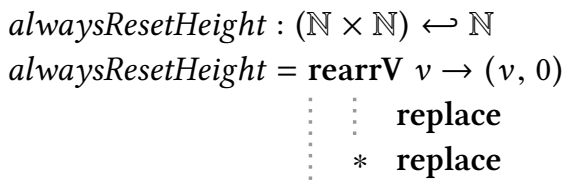

The two programs emb fst reset and alwaysResetHeight have roughly the same putback behaviour, which we can establish using putback triples. On the other hand, in the get direction, Theorem 3.8 can tell us that both $\mathcal{G}$ (get (emb fst reset)) and $\mathcal{G}$ (get alwaysResetHeight) are contained in $\mathcal{G}(\mathcal{F u s t} \circ f s t)$. But in fact, get (emb fst reset) can compute successfully on all inputs, whereas get alwaysResetHeight can only compute successfully on inputs whose second component is zero (and is usually not what one wants in practice). This reveals that we still lack the machinery for fully understanding forward behaviour. What we are missing is the ability to estimate the domain of a forward transformation, i.e., the subset of sources on which the forward transformation can compute successfully. To make such estimates for BIGUL programs, which describe putback transformations, the key insight is that, for a well-behaved pair of put and get, the domain of get coincides with the range of put, i.e., the subset of sources that can be produced by put. ${ }^{5}$ The problem with alwaysResetHeight is now clear: it can only produce the sources whose second component is zero, so get alwaysResetHeight can compute successfully only on those sources. By contrast, $e m b$ st reset is capable of producing all possible pairs. Our way ahead is to develop machinery for making such range estimates reliably, and that machinery is a second set of Hoare-style triples.

\subsection{Theory of Range Triples}

Definition 5.1. A range triple is a BIGUL program $b: S \hookleftarrow V$ surrounded by two range assertions: $\{\{R\}\} \quad b \quad\left\{\left\{P^{\prime}\right\}\right\}$

where $R: \mathcal{P}(S \times V)$ is the precondition or input range (on the original source and the view) and $P^{\prime}: \mathcal{P} S$ is the postcondition or output range (on the updated source). Valid range triples are inductively defined by the proof rules in Figure 3 (which will be explained in Section 5.2).

While the intended interpretation of a range triple for $b$ is about the range of put $b$, we actually need a slightly stronger interpretation about get $b$ (to make Theorem 5.4 work), as stated by the following soundness and completeness theorem (which is, in a sense, dual to Theorem 3.3).

Theorem 5.2 (soundness AND COMPLeteness of RANGe triples). Let $b: S \hookleftarrow V, R: \mathcal{P}(S \times V)$, and $P^{\prime}: \mathcal{P S}$.

$$
\{\{R\}\} \quad b \quad\left\{\left\{P^{\prime}\right\}\right\} \quad \text { if and only if } \forall s . P^{\prime} s \Rightarrow \exists v \text {. get } b s=\text { fust } v \wedge R s v .
$$

We can recover the intended interpretation of range triples by showing that the right-hand side of Theorem 5.2 is equivalent to a statement primarily about put, as stated in the following lemma.

LEMma 5.3. The right-hand side of Theorem 5.2 is equivalent to:

$$
\left(\forall s^{\prime} . P^{\prime} s^{\prime} \Rightarrow \exists s, v . R s v \wedge \text { put } b s v=\text { fust } s^{\prime}\right) \wedge \mathcal{G}(\text { get } b) \cap\left\langle s_{-} \mid P^{\prime} s\right\rangle \subseteq R
$$

The left conjunct in Lemma 5.3 is the primary way in which we think about the range triples: if $\{\{R\}\} b\left\{\left\{P^{\prime}\right\}\right\}$ can be derived, then the range of updated sources produced by applying put $b$ to those inputs satisfying $R$ will be at least $P^{\prime}$. The right conjunct in Lemma 5.3 says that there is an unintended "side effect" when we think about these triples in the putback direction: the input

\footnotetext{
${ }^{5}$ To see the coincidence, observe that PUTGET (as stated in Theorem 2.2) can be read roughly as "if $s^{\prime}$ is produced by put $b$, i.e., $s^{\prime}$ is in the range of put $b$, then get $b$ will compute successfully on $s^{\prime}$, i.e., $s^{\prime}$ will be in the domain of get $b^{\prime \prime}$, and GETPUT says the converse.
} 


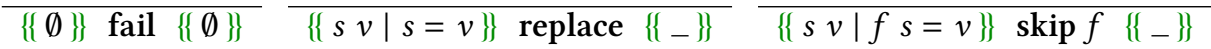

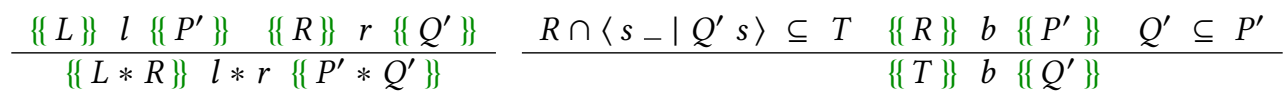

$$
\begin{aligned}
& \frac{\{\{s \text { wpat } \mid R s \overline{w p a t}\}\} \quad b \quad\left\{\left\{P^{\prime}\right\}\right\}}{\{\{s \text { vpat } \mid R s \overline{v p a t}\}\} \text { rearrV vpat } \rightarrow \text { wpat }\left\llcorner b\left\{\left\{P^{\prime}\right\}\right\}\right.}
\end{aligned}
$$

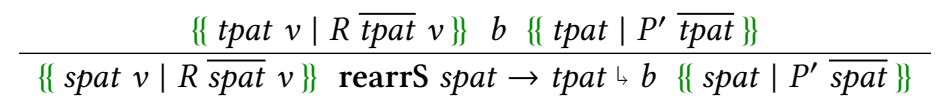$$
\forall n=(\text { normal } M \text { exit } E\llcorner b) \in b s \text {. }
$$

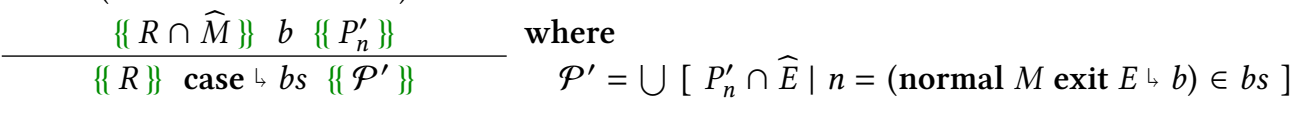

Fig. 3. Range proof rules

range considered will be forced to include those related by get with its domain restricted to $P^{\prime}$. So, for example, we will not be able to deduce $\{\{m n \mid m=n+1\}\}$ replace $\{\{-\}\}$ even though the left conjunct in Lemma 5.3 is true for this pair of $R$ and $P^{\prime}$. This "side effect" normally does not prevent us from deriving range triples, though, since preconditions are normally larger than consistency relations, which in turn contain the graphs of get transformations.

Back in Section 3, where we only had putback triples, Theorem 3.8 only enabled us to understand the forward behaviour of BIGUL programs to a limited extent. Now supplemented with range triples, we can prove a stronger and satisfactory result.

Theorem 5.4 (TOtAl Forward CONSISTEncy). Let $b: S \hookleftarrow V, R: \mathcal{P}(S \times V), C: \mathcal{P}(S \times V)$, and $P^{\prime}: \mathcal{P} S$.

$$
\begin{gathered}
\text { If }\{R\} b\left\{s^{\prime}-v \mid C s^{\prime} v\right\} \text { and }\{\{R\}\} b\left\{\left\{P^{\prime}\right\}\right\} \\
\text { then } \forall s . P^{\prime} s \Rightarrow \exists v \text {. get } b s=\text { fust } v \wedge C s v .
\end{gathered}
$$

Proof. Suppose that $P^{\prime}$ holds for a source $s$. By the range triple and Theorem 5.2, get $b s$ will compute successfully to some view $v$ such that $R s v$ holds, making $s$ and $v$ fall into $\mathcal{G}(g e t b) \cap R$. The putback triple and Theorem 3.8 can then take over and establish $C s v$ as required.

Theorem 5.4 tells us that, by supplementing a putback triple for $b$ with a range triple with the same precondition, we can know on which subset of sources get $b$ will compute successfully and that the behaviour of get $b$ will conform to the consistency relation stated in the putback triple.

In summary, now we have enough machinery to tell us all we want to know about the bidirectional behaviour of a BIGUL program: By deriving a putback triple $\{R\} b\left\{s^{\prime} s v \mid C s^{\prime} v \wedge R^{\prime} s^{\prime} s v\right\}$ to reason about the behaviour of $b$, we know that put $b$ will compute successfully on $R$, establish consistency $C$, and have retentive behaviour $R^{\prime}$. Then, by additionally deriving a range triple $\{\{R\}\} b\left\{\left\{P^{\prime}\right\}\right\}$ to estimate the range of $b$, we know that get $b$ will compute successfully on $P^{\prime}$ and conform to the same consistency relation $C$ established by put $b$. Notably, as we will see next, derivations of range triples are usually significantly easier than derivations of putback triples, so in practice there is usually not much more work to do than deriving putback triples. 


\subsection{The Range Proof Rules}

The range proof rules are shown in Figure 3. The most interesting rule is probably the consequence rule (the right one in the second row), whose direction is just the opposite of the putback consequence rule given in Figure 2. An explanation is that the ultimate interpretation of range triples, as stated by Theorem 5.2, is about forward behaviour, and the output/input range in a range triple in fact serves the role of precondition/postcondition for the forward transformation. But, interestingly, the consequence rule can also be understood in the putback direction: If $\{\{R\}\} b\left\{\left\{P^{\prime}\right\}\right\}$ has been established, meaning that the inputs in $R$ can induce everything in $P^{\prime}$ through the execution of $b$, then a larger input range $T$ can still induce everything in $P^{\prime}$, or indeed everything in any output range $Q^{\prime}$ smaller than $P^{\prime}$. In fact, $T$ is not necessarily larger than $R$ : if what we eventually target is a smaller output range, then we will be allowed to also shrink the input range - as stated in the consequence rule, we are allowed to use $Q^{\prime}$ to constrain the sources in the input range. Since the direction of the range consequence rule is the opposite of the putback one, when deriving a range triple using the consequence rule in our derivation format, logical implications go upwards, and we can use the postconditions for a node as additional premises when proving implications between preconditions for the same node, opposite to what we do in putback derivations.

Other rules should be largely intuitive. The fail rule has the empty predicate as its output range since fail can never produce anything (and its input range can be any relation because of the consequence rule). The replace rule says that replace can produce everything as long as the input range is large enough - because of the "side effect" explained below Lemma 5.3, the input range has to be large enough to include the graph of replace's forward semantics, which is the identity transformation. The skip rule has the same precondition as its putback counterpart and says that skip can produce everything. The product and rearrangement rules are analogous to their putback counterparts. For case, only normal branches matter since execution of case always ends in a normal branch. We estimate an output range $P_{n}^{\prime}$ for the body of every normal branch $n$, and the estimated output range for that branch is $P_{n}^{\prime}$ intersected with the actual exit condition, since only outputs satisfying the actual exit condition can be produced. The estimated output range for the entire case is then the union of the estimated output ranges for all the normal branches.

Example 5.5 (embedding pairs of transformations in BIGUL). As we mentioned, derivations of range triples can be very straightforward in simple cases. In the case of emb, for example, we can effortlessly prove that it produces everything:

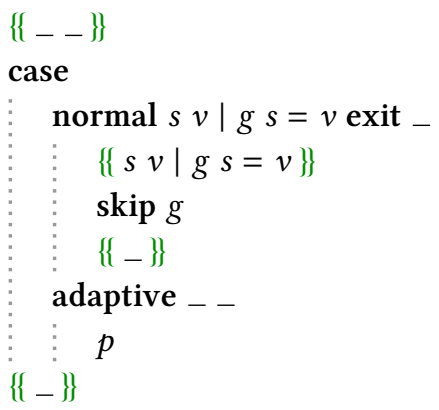

Example 5.6 (rectangle width updating - always resetting the height). Verifying the range of alwaysResetHeight is a more interesting example, where we will see how a non-trivial output range can be derived with the help of the consequence rule:

$\{\{--\}\}$

rearrV $v \rightarrow(v, 0)$ 


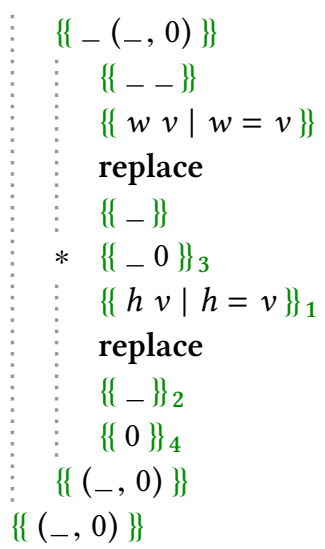

The interesting part is the second replace, for which we first establish the input and output ranges as assertions 1 and 2 according to the replace rule. However, because of the outer rearrV, the views in the actual input range for the second replace are restricted to zero, as stated by assertion 3, which does not contain assertion 1 . We therefore need to shrink our estimate of the output range of replace to just zero (assertion 4) to allow us to also restrict the views in the input range to zero. Logically, assertions 1 and 4 together indeed imply assertion 3, adhering to the consequence rule.

\section{RECURSION}

BIGUL is designed datatype-generically [Gibbons 2007] to work with inductive data structures. A lot of recursive programs processing inductive data have been written using the HASKELL port of BIGUL, and our Hoare-style logic would not be useful at all if we could not reason about such recursive BIGUL programs. For example, let us take a peek at the key-based list alignment program keyAlign in Figure 4, which we will verify in Section 7. All we need to care about in regard to keyAlign now is its recursive structure. Both the source and view are lists, and in the second branch of the case, they are both non-empty. Inside the branch, each of them is rearranged into a pair of its head and tail, and we recursively invoke the program to process the tails. Intuitively, we know that this program terminates for any input because the size of the initial source and view is strictly larger than the size of the source and view at the point of the recursive invocation. We will need to incorporate this size-based termination argument into our proof rules for recursive programs, and show that the rules are sound.

There is difficulty dealing with recursive programs in the AGDA formalisation underlying this paper, though. Observe that the program structure of keyAlign is infinite, which is fine in HASKELL; in the AGDA formalisation, however, BIGUL programs are modelled inductively and are necessarily finite. One possible solution is to redefine BIGUL programs coinductively and bring in the partiality monad [Capretta 2005] to model non-termination in HASKELL, but this means abandoning most (if not all) of the previous formalisation effort. Another possible solution is to stay with inductive BIGUL programs and introduce a "terminating fixed-point" which can decide, for every input, how many times the body of a fixed-point should be expanded, thereby circumventing the modelling of infinite program structures. This approach will be relevant in a constructive setting, but we anticipate that there will be extra constructivity requirements that are not relevant for the HASKELL port of BIGUL, in which most (recursive) BIGUL programs are written. Since what we aim at in this paper is not thorough formalisation but a semi-formal reasoning framework for the working BIGUL programmer, we will develop just enough theory to justify our rules for reasoning about recursive programs, and refrain from delving into coinductiveness or constructive termination. 
Let $b: S \hookleftarrow V$ be a recursive program of the form $b=f b$ where $f:(S \hookleftarrow V) \rightarrow(S \hookleftarrow V)$ is the usual non-recursive function defining the body of $b$, and suppose that we want to verify that $b$ can successfully turn any input satisfying a precondition $R$ into an output satisfying a postcondition $R^{\prime}$. We cannot hope to establish $\{R\} b\left\{R^{\prime}\right\}$ (although we will abuse this notation in Section 7) since putback triples are defined for finite programs, whereas $b$ is infinite. Instead, when we say in this paper that we are verifying $b$, what we precisely mean is verifying the behaviour of all finite expansions of its body $f$, where finite expansions are defined by:

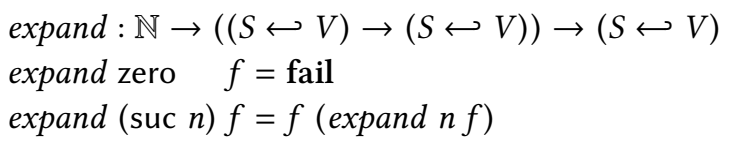

The basic idea is to prove something about $f$ like:

$\forall$ rec $: S \hookleftarrow V . \quad\{R\} \operatorname{rec}\left\{R^{\prime}\right\} \Rightarrow\{R\}$ frec $\left\{R^{\prime}\right\}$

which can then be iterated to produce $\{R\}$ expand $n f\left\{R^{\prime}\right\}$ for any $n$ - the base case is $\{R\}$ fail $\left\{R^{\prime}\right\}$, which implies $\{R\} f$ fail $\left\{R^{\prime}\right\}$, and then $\{R\} f$ ( $f$ fail) $\left\{R^{\prime}\right\}$, etc. This idea cannot be directly valid, however, since in general only a subset of $R$ can be successfully processed by a finite expansion of $f$ (whose execution can be thought of as executing $b$ but allowing recursive invocations only to a certain depth). We thus introduce a function of type $S \rightarrow V \rightarrow \mathbb{N}$ for measuring the size of the source and view in assertions, and include size restrictions in the preconditions for the finite expansions, leading to the following theorem.

TheOREM 6.1 (Finite EXPANSION OF PUtBACK TRIPLES). Let $f:(S \hookleftarrow V) \rightarrow(S \hookleftarrow V)$, $R: \mathcal{P}(S \times V), R^{\prime}: \mathcal{P}(S \times S \times V)$, and measure $: S \rightarrow V \rightarrow \mathbb{N}$. If

$\forall n$, rec. $\quad(\forall m . \quad\{R \cap\langle s v|$ measure $\left.s v=m \wedge m<n\rangle\} \operatorname{rec}\left\{R^{\prime}\right\}\right)$

$$
\Rightarrow \quad\{R \cap\langle s v| \text { measure } s v=n\rangle\} f \operatorname{rec}\left\{R^{\prime}\right\}
$$

(PutbackRecursion)

then:

$\forall l, n . n \leqslant l \Rightarrow\{R \cap\langle s v|$ measure $s v=n\rangle\}$ expand (suc $l) f\left\{R^{\prime}\right\}$

PutbackRecursion will be the proof rule we use for reasoning about the putback behaviour of recursive programs. It combines the basic proof idea (3) with the size-based termination argument given in the beginning of this section: in the precondition for $f$ rec, the size of the input source and view is bound to a logic variable $n$, and we can make recursive invocations wherever the size $m$ of the current source and view is strictly less than $n$. The soundness of PutbackRecursion is justified by Theorem 6.1, whose conclusion implies that any input in $R$ can be successfully turned into an output in $R^{\prime}$ as long as $f$ is expanded enough times.

Analogously, we have a RANGERECURSION rule for estimating the output ranges of recursive programs.

Theorem 6.2 (Finite EXPANSion of RANGe triples). Let $f:(S \hookleftarrow V) \rightarrow(S \hookleftarrow V), R: \mathcal{P}(S \times V)$, $P^{\prime}: \mathbb{N} \rightarrow \mathcal{P} S$, and measure $: S \rightarrow V \rightarrow \mathbb{N}$. If

$$
\begin{gathered}
\left.\forall n, \text { rec. } \quad(\forall m . \quad\{\{R \cap\langle s v| \text { measure } s v=m\rangle\}\} \operatorname{rec}\left\{\left\{P^{\prime} m \cap\langle-\mid m<n\rangle\right\}\right\}\right) \\
\Rightarrow \quad\{\{R \cap\langle s v| \text { measure } s v=n\rangle\}\} \text { frec }\left\{\left\{P^{\prime} n\right\}\right\} \quad \text { (RangeRecursion) }
\end{gathered}
$$

then:

$$
\forall l, n . n \leqslant l \Rightarrow\{\{R \cap\langle s v| \text { measure } s v=n\rangle\} \text { expand (suc } l) f\left\{\left\{P^{\prime} n\right\}\right\}
$$




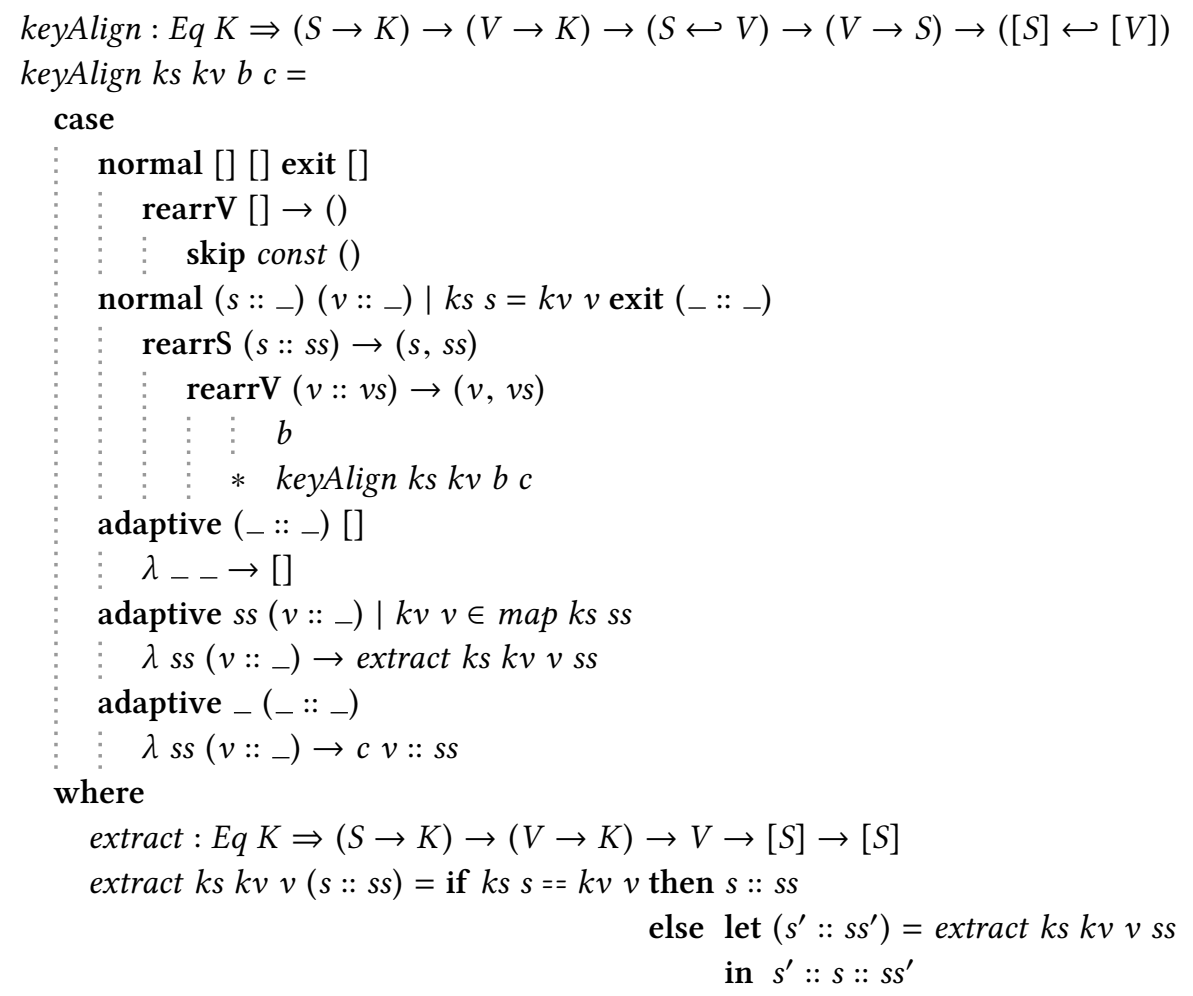

Fig. 4. Key-based list alignment in BıGUL

The RangeRecursion rule instructs us to derive an output range $P^{\prime} n$ that can depend on the logic variable $n$ bound to the size of the input source and view in the precondition. (For example, the range triple we will derive for keyAlign is $\{\{\ldots$ s $\mid$ length $v s=n\}\}$ keyAlign $\ldots .\{\{s s \mid$ length $s s=n\}\}$.) Recursive invocations can be made in the derivation, and the estimated output range for a recursive invocation is $P^{\prime} m$ where $m$ is the size of the current source and view, provided that $m$ is strictly less than $n$ - otherwise, the estimated output range will be empty. The conclusion of Theorem 6.2 justifies the soundness of RANGERECURSION, as it implies that every output in $\bigcup_{n: \mathbb{N}} P^{\prime} n$ can be produced from some input of the right size in $R$ as long as $f$ is expanded enough times.

Having the two recursion rules, we are now ready to verify keyAlign.

\section{VERIFYING KEY-BASED LIST ALIGNMENT}

Alignment is a representative problem for bidirectional transformations [Bohannon et al. 2008; Barbosa et al. 2010; Diskin et al. 2011; Pacheco et al. 2012; Voigtländer et al. 2013; McKinna 2016]. In this paper, we focus on the specialised (and asymmetric) setting where both the source and view are lists, which are consistent exactly when they have the same length and an element-level consistency relation is satisfied by each pair of the source and view elements at the same position. View elements may be inserted, deleted, modified, or reordered. To put the updated view list back into the source list, we need to align the two lists, i.e., decide for each view element to which source element it corresponds (if any), before we can invoke an element-level consistency restorer on the right pairs of source and view elements. 
Several variants of list alignment have been implemented in BIGUL [Zan et al. 2016; Mendes et al. 2016]. In this paper we choose to verify a variant that is non-trivial and yet not overly complicated: key-based alignment, of which an implementation was presented and explained with a concrete scenario by $\mathrm{Hu}$ and Ko [2017, Section 6.2]. Their program keyAlign is shown in Figure 4. The types of source and view elements are $S$ and $V$ respectively. The program takes two functions $k s: S \rightarrow K$ and $k v: V \rightarrow K$ as arguments, which are used to extract a key value of type $K$ from every source or view element, and the type $K$ should support decidable equality. Two more arguments $b$ and $c$ are needed to deal with two of the three possible situations that can result from an alignment:

- A view element $v$ is deemed to correspond to a source element $s$ only if their keys match, i.e., $k s s=k v v$; an element-level synchroniser $b: S \hookleftarrow V$ will then be invoked on this pair of source and view elements.

- If no source element has the same key as a view element, a function $c: V \rightarrow S$ will be used to create a temporary corresponding source; this temporary source will then be fully synchronised with the view using $b$.

- A source element will be deleted if there is no corresponding view element.

Here is a quick overview of the program: The first branch is the base case. The second branch deals with "happy coincidences": the head elements in the source and view lists match, so we can simply synchronise the heads and recursively process the tails. The third branch deletes everything in the source when the view is empty. The fourth branch is the most interesting: the head view element $v$ has a corresponding source element (which is not at the head position), so we extract the first source element with the same key as $v$ and put it at the head (intending to re-enter the second branch afterwards). When there is no source element corresponding to the head view element, the fifth and last branch uses $c$ to create a temporary corresponding source element. Note that the program uses some partial functions, which are fine in HAskell but not in AgDA: extract, for example, misses two cases for empty source lists, and these cases have to be added for verification in AgdA. These missing cases are irrelevant, however, since keyAlign does not invoke extract on empty source lists.

To verify keyAlign, we need to make some assumptions about its arguments. For simplicity, we assume that $b$ can compute successfully for any pair of source and view elements with the same key; also, $b$ should guarantee that the updated source and the view will have the same key, apart from any other postcondition $R^{\prime}: \mathcal{P}(S \times S \times V)$ it can establish. As a putback triple:

$$
\{s v \mid k s s=k v v\} \quad b \quad\left\{R^{\prime} \cap\left\langle s^{\prime}-v \mid k s s^{\prime}=k v v\right\rangle\right\}
$$

We will abbreviate $R^{\prime} \cap\left\langle s^{\prime}-v \mid k s s^{\prime}=k v v\right\rangle$ as $T^{\prime}$. For the source-creating function $c$, since a created source will be further processed by $b$, we require the key of the created source to be the same as that of the view:

$$
\forall v . k s(c v)=k v v
$$

We can then derive, for all $n$ :

$$
\left\{{ }_{-} v s \mid \text { length } v s=n\right\} \text { keyAlign ks } k v b c\left\{s s^{\prime} s s v s \mid \exists \widetilde{s s} . T^{\prime \star} s s^{\prime} \widetilde{s s} v s \wedge \text { Retentive ss vs } \widetilde{s s}\right\}
$$

In the precondition, we use the length of the view list as the termination measure, but otherwise impose no restrictions on the source and view lists. In the postcondition, the relation $T^{\prime \star}: \mathcal{P}([S] \times[S] \times[V])$ is defined inductively by the following two rules:

$$
\begin{array}{lll}
T^{\prime \star}[] & {[]} & {[]} \\
T^{\prime \star}\left(s^{\prime}:: s s^{\prime}\right)(\tilde{s}:: \widetilde{s s})(v:: v s) \Leftarrow T^{\prime} s^{\prime} \tilde{s} v \wedge T^{\prime \star} s s^{\prime} \widetilde{s s} v s
\end{array}
$$


The postcondition thus guarantees that the updated source list $s s^{\prime}$ will have the same length as $v s$, and for each pair of source element $s^{\prime}$ in $s s^{\prime}$ and view element $v$ in $v s$ at the same position, $T^{\prime}$ will be established for $s^{\prime}$, some source element $\tilde{s}$, and $v$. The elements $\tilde{s}$ are collected into a list $\widetilde{s s}$, and Retentive ss vs $\widetilde{s s}$ says that $\widetilde{s s}$ contains those source elements in ss that correspond to some view element in $v s$. This Retentive relation turns out to be slightly tricky to define, especially when we do not require that keys in a list are all unique; our definition says that if a key appears $n$ times in the view list, then the first $n$ elements with that key in the original source list will be retained.

Due to space restrictions, we only sketch the verification of the second normal branch and the second adaptive branch. The assertions for the second normal branch are as follows, where we omit the invocations of the rearrS, rearrV, and product rules since they are straightforward in this case:

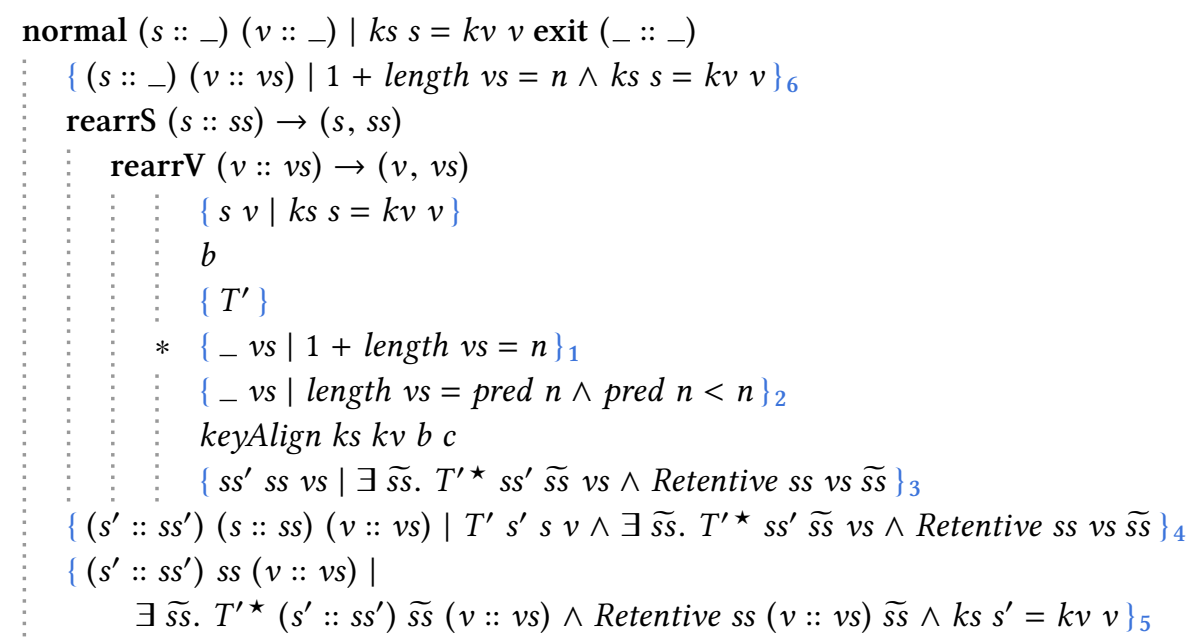

We first look at how the PutbackRecursion rule is applied. Assertion 1 is the actual precondition for the recursive invocation, and we rewrite it into assertion 2, saying that the length of the view list at this point is pred $n$, which is strictly less than $n$ since assertion 1 says that $n$ is a successor. (The predecessor function is defined by pred zero = zero and pred (suc $n$ ) $=n$, so pred $n$ is not necessarily less than $n$.) The recursive invocation will thus succeed and establish assertion 3 . For the postconditions, the rearrS, rearrV, and product rules give us assertion 4, while we need to prove assertion 5. The last conjunct $k s s^{\prime}=k v v$ in assertion 5 is part of $T^{\prime} s^{\prime} s v$ in assertion 4 by definition. From $T^{\prime} s^{\prime} s v$ and $\exists \widetilde{s s}$. $T^{\prime \star} s s^{\prime} \widetilde{s s} v s$ in assertion 4, we see that we should use $s:: \widetilde{s s}$ as the new $\widetilde{s s}$ in assertion 5 . We are left to prove Retentive $(s:: s s)(v:: v s)(s:: \widetilde{s s})$ (since $s s$ in assertion 5 is $s::$ ss in assertion 4), which is implied by Retentive ss $v s \widetilde{s s}$ in assertion 4 and $k s=k v v$ in assertion 6 .

Now we turn to the second adaptive branch:

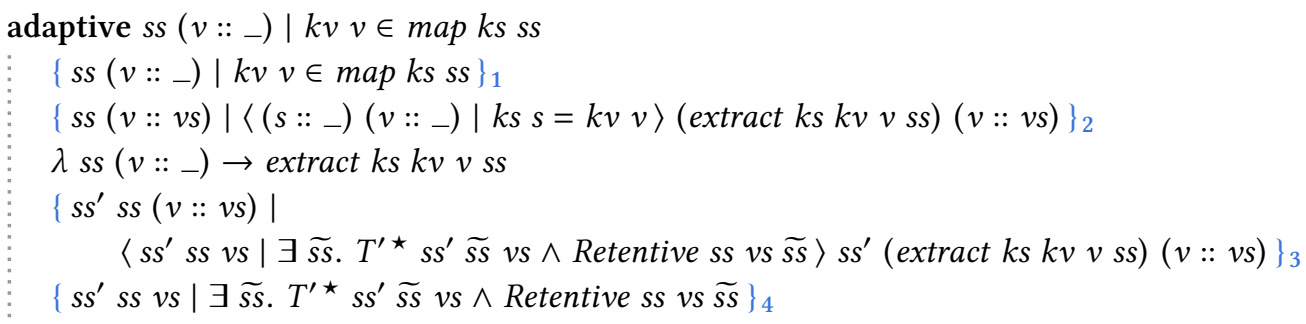

Assertion 1 (where we omit the negations of the previous main conditions) implies assertion 2, which is the condition for re-entering the second normal branch: the source list ss must be non-empty 
so that extract $k s k v v s s$ will successfully produce a non-empty list, whose head will then have the same key as the head view element $v$. After adaptation, the rerunning of the case establishes assertion 3, which should be shown to imply assertion 4, the final postcondition. Assertion 3 says that $T^{\prime \star}$ holds for the updated source list $s s^{\prime}$, some list $\widetilde{s s}$ of source elements, and the view list, and we can directly use $\widetilde{s s}$ as the witness and establish the first conjunct in assertion 4 . Assertion 3 also says that $\widetilde{s s}$ retains certain elements from the adapted source list, which is just the original source list with its first source element having key $k v v$ moved to the head position, so we know that $\widetilde{s s}$ retains the same elements in the original source list as well. This is an illustrative example showing that adaptation should be done cautiously to maintain sufficient similarity between the adapted source and the original source, or otherwise it can be immensely difficult to prove the implication from the adapted postcondition to the final postcondition.

We should not forget to derive a range triple for keyAlign. For simplicity, let us derive:

$\forall n . \quad\{\{-v s \mid$ length $v s=n\}\}$ keyAlign $k s k v b c \quad\{\{s s \mid$ length $s s=n\}\}$

assuming:

$\{\{s v \mid k s s=k v v\}\} \quad b \quad\{\{-\}\}$

That is, if $b$ is capable of producing everything, then keyAlign $k s k v b c$ can also produce everything. If $T^{\prime} \subseteq\left\langle s^{\prime}-v \mid C s^{\prime} v\right\rangle$ for some element-level consistency relation $C: \mathcal{P}(S \times V)$, then by Theorem 5.4 we know that for any source list, get (keyAlign $k s k v b c$ ) will successfully produce a view list of the same length, and each pair of source and view elements at the same position will satisfy $C$. For the derivation, again due to space restrictions we can only give a quick sketch: We only need to derive ranges for the two normal branches. The output range of the first branch can be derived as $\langle[] \mid 0=n\rangle-$ that is, the branch can produce empty lists when $0=n$; as for the output range of the second branch, we can derive $\langle(-:: s s)| 1+$ length $s s=n\rangle$. Their union is the output range of the entire case, and is indeed $\langle s s|$ length $s s=n\rangle$.

\section{DISCUSSION}

How expressive is BIGUL (especially compared with existing languages)? The expressive power of the version of BIGUL used in this paper mainly stems from its case construct, which has gone beyond Foster et al.'s [2007] "general conditional" and allows, in particular, key-based list alignment to be implemented using only simple and general-purpose primitives for the first time. (In the original BIGUL [Ko et al. 2016], the case analysis constructs were essentially the same as Foster et al.'s conditionals, and key-based list alignment had to be provided as an extra and complex primitive.)

Regarding alignment, Barbosa et al.'s [2010] "matching lenses" offer several sophisticated matching strategies, some of which can be hard to implement "nicely" in BIGUL so far. However, matching lenses are special-purpose and require the invention of dedicated laws, and the essential components are built from scratch, whereas BIGUL is designed with the ultimate aim of expressing all lenses using just a fixed set of simple primitives, like what we can do in general-purpose languages. In particular, we can program alignment in BIGUL without having to bake special-purpose concepts like Barbosa et al.'s "chunks", "rigid complements", "resources" etc into the language.

While seemingly simple, BIGUL has been successfully employed in several practical scenarios, including web server configuration adaptation [Colson et al. 2016], parsing and reflective printing [Zhu et al. 2016], synchronisation of feature configurations and use cases [Zhao et al. 2016], and synchronisation of executable programs and proof scripts [Kinoshita and Nakano 2017].

BIGUL claims to be "putback-based" but is still a lens language. Is there really a fundamental difference between BIGUL and previous "get-based" lens languages? Regarding language definition, 
BIGUL programs denote lenses and have to be defined in both directions, exactly the same as other lens languages. It is when it comes to using the lenses that the distinction between the getand put-based approaches becomes meaningful. The majority of lens languages are get-based, as explained by Foster [2009] below his Lemma 2.2.6: 'Lens programmers often feel like they are writing the forward transformation (because the names of primitives typically connote the forward transformation) and getting the backward transformation "for free" '. Matsuda and Wang [2015], for example, explicitly state that their language adopts this design. Foster et al. [2007] also clearly show in their Figure 8 that their lens programs are supposed to be constructed like writing get, and Bohannon et al.'s [2006] relational lenses are written like database queries, which are get transformations. Their programs can be (and are usually) enriched with putback information to allow more control, but that makes constructing and understanding the programs more awkward (see the next paragraph). By contrast, BIGUL's putback-based design lets the programmer construct programs purely in the put direction. The Hoare-style logic helps to clearly distinguish the two approaches for the first time: it is possible to precisely reason about bidirectional behaviour purely in the put direction, whereas it is unthinkable that the same can be achieved in the get direction. This might explain that there is only one comparable (but still much less powerful) reasoning framework: the totality lemmas of Foster et al. [2007], which can only establish properties equivalent to triples of the form $\{\{--\}\} b\{\{P\}\}$ and $\{s v \mid P s \wedge Q v\} b\left\{-_{-}\right\}$where $P: \mathcal{P} S$ and $Q: \mathcal{P} V$.

Didn't some get-based approaches also offer the ability to control putback behaviour? Why switch to the putback-based approach? To name a few, the "fixup functions" in Foster et al.'s [2007] "general conditionals" (for branch switching), the parameters of Bohannon et al.'s [2005] join_template (for resolving ambiguous deletions), and the alignment keywords like "key" and "best" in the BoomerANG language [Bohannon et al. 2008; Barbosa et al. 2010] (for specifying keys and matching strategies during alignment) are all constructs which appear in programs designed to look like forward transformations but are meaningful only in the putback direction. Constructs with similar purposes can also be found in bidirectionalisation approaches, such as Voigtländer et al.'s [2013] "shape bidirectionaliser plug-ins" (for programming shape changes). Apart from offering only limited and/or special-purpose customisation of putback behaviour, the fundamental problem with these languages is that their programs contain an ad hoc mixture of forward and backward information, and to properly understand such programs, the only way is to reason in both directions and in terms of the complex underlying semantics. In other words, it is hard to come up with easy-to-use reasoning principles for these languages, and since reasoning principles reflect and even guide how we program, this indicates that these languages fail to deliver an easy-to-use abstraction. BIGUL is unique since it offers a successful abstraction in which bidirectional programs become unidirectional and can still be precisely reasoned about, as clearly reflected in the Hoare-style logic.

The semantics of range triples (Theorem 5.2) is about the get direction; consequently, doesn't Theorem 5.4 say that we need to reason in both directions anyway, undermining the claim that putback-based reasoning is sufficient? Range triples are used only for establishing the domain of get - even without a range triple, a strong enough putback triple alone (i.e., one whose precondition is always true) can already imply that the get behaviour is constrained by the consistency relation (Theorem 3.8). And, starting from Lemma 5.3, we have explained how range triples can be understood and derived by thinking in the putback direction, without having to introduce the get semantics (except for the precondition of replace, which is only a minor exception though); this is particularly evident in the case range rule, which is much more awkward to interpret in the get direction. Even if a sceptical reader insisted on thinking about range triples in the get direction, it would still be much easier to prove that get is contained in the precondition for put (as required by Theorem 5.4) than to prove that it is contained in the consistency relation, which is usually much smaller than the 
precondition for put. The indisputable fact is that the major work is done in derivations of putback triples, making the reasoning putback-based.

Where is lens composition? In terms of consistency, the behaviour of lens composition is just relational composition; on the other hand, the retentive behaviour of lens composition is rather chaotic and hard to reason about, because its put direction is defined in terms of both the put and get directions of the lenses being composed. We can formulate a rule like:

$$
\begin{aligned}
& \left\{a b^{\prime} \mid \exists b, c . R a c \wedge R^{\prime} a b \wedge U^{\prime} b^{\prime} b c\right\} \quad l \quad\left\{\left\langle a^{\prime} b_{-} b \mid R^{\prime} a^{\prime} b\right\rangle \cap T^{\prime}\right\} \\
& \left\{\left\{a b^{\prime} \mid \exists b, c . R a c \wedge R^{\prime} a b \wedge U^{\prime} b^{\prime} b c\right\}\right\} l\left\{\left\{P^{\prime}\right\}\right\} \\
& \frac{\left\{b c \mid \exists a . R^{\prime} a b \wedge R a c\right\} r\left\{U^{\prime}\right\}}{\left\{R \cap\left\langle a_{-} \mid P^{\prime} a\right\rangle\right\} l \circ r\left\{a^{\prime} a c \mid \exists b, b^{\prime} . T^{\prime} a^{\prime} a b^{\prime} \wedge U^{\prime} b^{\prime} b c\right\}}
\end{aligned}
$$

and we have actually proved that the rule is sound, but the form of the rule is too complex to be easily usable. We will need to find a sweet spot and design a composition rule that is perhaps not as general as the above one but can still say enough about the retentive behaviour; most importantly, this rule should give guidance on how composition can be used and reasoned about in practice. It should be noted that while composition is included in other languages like Foster et al.'s [2007] original lenses (and in fact the HASKeLL port of BIGUL), the problem with controlling the retentive behaviour of composition has always existed, as discussed by, e.g., Diskin et al. [2011, Section 2.2].

Are there more examples of verified BIGUL programs? In the supplementary AGDA code, there is one more program replaceAll which replaces all the elements in a source list with a view:

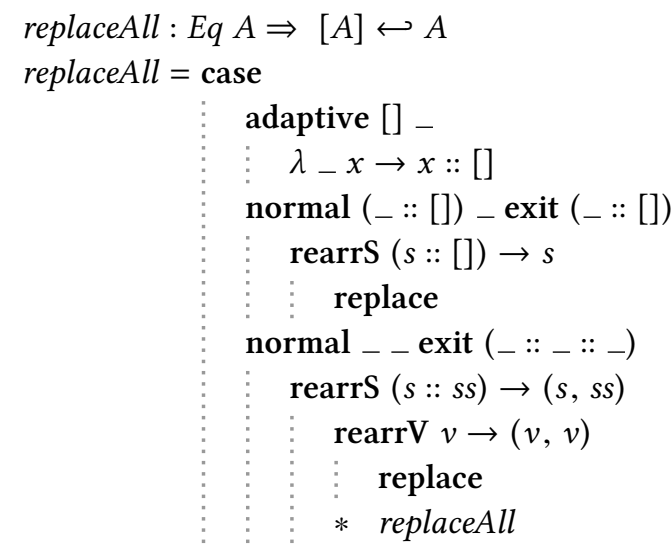

for which the following triples are verified:

$\forall n . \quad\left\{s s_{-} \mid\right.$length $\left.s s=n\right\} \quad$ replaceAll

$$
\left\{s s^{\prime} s s v \mid\left(\forall t . t \in s s^{\prime} \Rightarrow t=v\right) \wedge\left(s s \neq[] \Rightarrow \text { length } s s^{\prime}=\text { length } s s\right)\right\}
$$

$\forall n . \quad\{\{s s+\mid$ length $s s=n\}\} \quad$ replaceAll $\quad\left\{\left\{\left(s^{\prime}:: s s^{\prime}\right) \mid 1+\right.\right.$ length $\left.\left.s s^{\prime}=n \wedge \forall t . t \in s s^{\prime} \Rightarrow s^{\prime}=t\right\}\right\}$

This example helps to clarify the misconception that, when programming put, the programmer still needs to have a get in mind - rather, what the programmer needs to have in mind is a consistency relation ( $\left\langle s s^{\prime} v \mid \forall t . t \in s s^{\prime} \Rightarrow t=v\right\rangle$ in this case), which may be functional but does not need to be executable. The range triple is also interesting as it has a non-trivial output range.

Rather than developing more examples at this stage, we plan to move forward and aim for the verification of practical bidirectional applications. This will require better mechanised support than the current AGDA formalisation, which is exceedingly tedious to work with. We plan to adapt the 
Hoare-style logic for automated theorem proving (perhaps in the style of LiQUidHASKELL [Vazou et al. 2014]), so as to verify larger-scale bidirectional programs with reasonable effort.

\section{CONCLUSION}

Based on Lemma 2.3, it has been argued that "putback" is the essence of bidirectional programming [Fischer et al. 2015b]. We would like to amend this statement: putback-based reasoning is the essence of bidirectional programming. With the Hoare-style logic for BIGUL, we have demonstrated how we can understand a BIGUL program's bidirectional behaviour by reasoning exclusively about its putback behaviour, reducing bidirectional programming to unidirectional programming.

Bidirectional programming has been based on a declarative model, in which the programmer writes a consistency specification and relies on the system to produce a well-behaved implementation, whose consistency restoration behaviour can be customised to varying extents but usually in ad hoc and/or awkward ways. However, it has long been realised that declarative approaches are hardly enough for practical bidirectional applications (see, e.g., Stevens [2010, Section 4.1]). The bidirectional transformations community currently concentrates on the exploration of more forms of well-behavedness laws (see, e.g., Cheney et al. [2017]), but we should not be satisfied with only well-behavedness guarantees. Instead, we should also start aiming to precisely characterise the behaviour of bidirectional programs like what the BIGUL programmer can now do with the Hoare-style logic, and only then can we think about more complex bidirectional applications and the verification of their consistency restoration behaviour.

More broadly, we believe that programming languages should be shipped with reasoning principles - even domain-specific languages deserve domain-specific reasoning principles, to justify that the languages offer adequate abstractions, and to help the programmer to work effectively and reliably with those abstractions. In the case of BIGUL, the Hoare-style logic reflects the somewhat stateful nature of BIGUL programming, and is designed domain-specifically such that the programmer can work out the precise behaviour of BIGUL programs with reasonable effort (rather than breaking the abstraction and working with the messier underlying semantics). Moreover, the evolution of BIGUL is partly prompted by the development of the Hoare-style logic, whose eventual simplicity justifies BIGUL's current design. If, as Dijkstra [1974] argued, programs and their correctness proofs should grow hand in hand, then programming languages and their reasoning principles ought to be developed together as well. BIGUL and its Hoare-style logic make a nice example of this statement.

\section{ACKNOWLEDGEMENTS}

We would like to thank Jeremy Gibbons, Li Liu, and Zirun Zhu for commenting on drafts of this paper, Shin-Cheng Mu for the fruitful discussions during his visit at NII, and Zhixuan Yang for proofreading the manuscript. We also thank the anonymous reviewers for their valuable comments, and our shepherd James Cheney for checking a nearly final version of this paper. This work is partially supported by the Japan Society for the Promotion of Science (JSPS) Grant-in-Aid for Scientific Research (A) No. 25240009 and (S) No. 17H06099.

\section{REFERENCES}

Davi M. J. Barbosa, Julien Cretin, Nate Foster, Michael Greenberg, and Benjamin C. Pierce. 2010. Matching Lenses: Alignment and View Update. In International Conference on Functional Programming (ICFP'10). ACM, 193-204. https: //doi.org/10.1145/1863543.1863572

Aaron Bohannon, J. Nathan Foster, Benjamin C. Pierce, Alexandre Pilkiewicz, and Alan Schmitt. 2008. Boomerang: Resourceful Lenses for String Data. In Symposium on Principles of Programming Languages (POPL'08). ACM, 407-419. https://doi.org/10.1145/1328897.1328487 
Aaron Bohannon, Benjamin C. Pierce, and Jeffrey A. Vaughan. 2005. Relational Lenses: A Language for Updatable Views. Technical Report. Department of Computer and Information Science, University of Pennsylvania. http://www.cis.upenn. edu/ bcpierce/papers/dblenses-tr.pdf

Aaron Bohannon, Benjamin C. Pierce, and Jeffrey A. Vaughan. 2006. Relational Lenses: A Language for Updatable Views. In Symposium on Principles of Database Systems (PODS'06). ACM, 338-347. https://doi.org/10.1145/1142351.1142399

Venanzio Capretta. 2005. General Recursion via Coinductive Types. Logical Methods in Computer Science 1, 2 (2005), 1-28. https://doi.org/10.2168/LMCS-1(2:1)2005

James Cheney, Jeremy Gibbons, James McKinna, and Perdita Stevens. 2017. On Principles of Least Change and Least Surprise for Bidirectional Transformations. Journal of Object Technology 16, 1 (2017), 3:1-31. https://doi.org/10.5381/jot.2017.16.1.a3

Kevin Colson, Robin Dupuis, Lionel Montrieux, Zhenjiang Hu, Sebastián Uchitel, and Pierre-Yves Schobbens. 2016. Reusable Self-Adaptation through Bidirectional Programming. In International Symposium on Software Engineering for Adaptive and Self-Managing Systems (SEAMS'16). ACM, 4-15. https://doi.org/10.1145/2897053.2897055

Krzysztof Czarnecki, J. Nathan Foster, Zhenjiang Hu, Ralf Lämmel, Andy Schürr, and James F. Terwilliger. 2009. Bidirectional Transformations: A Cross-Discipline Perspective. In International Conference on Model Transformation (Lecture Notes in Computer Science), Vol. 5563. Springer, 260-283. https://doi.org/10.1007/978-3-642-02408-5_19

Edsger W. Dijkstra. 1974. Programming as a Discipline of Mathematical Nature. Amer. Math. Monthly 81, 6 (1974), 608-612. https://doi.org/10.2307/2319209

Zinovy Diskin, Yingfei Xiong, and Krzysztof Czarnecki. 2011. From State- to Delta-Based Bidirectional Model Transformations: the Asymmetric Case. Journal of Object Technology 10 (2011), 6:1-25. https://doi.org/10.5381/jot.2011.10.1.a6

Sebastian Fischer, Zhenjiang Hu, and Hugo Pacheco. 2015a. A Clear Picture of Lens Laws. In International Conference on Mathematics of Program Construction (Lecture Notes in Computer Science), Vol. 9129. Springer, 215-223. https: //doi.org/10.1007/978-3-319-19797-5_10

Sebastian Fischer, Zhenjiang Hu, and Hugo Pacheco. 2015b. The Essence of Bidirectional Programming. SCIENCE CHINA Information Sciences 58, 5 (2015), 1-21. https://doi.org/10.1007/s11432-015-5316-8

John Nathan Foster. 2009. Bidirectional Programming Languages. Ph.D. Dissertation. University of Pennsylvania. http: //repository.upenn.edu/edissertations/56

J. Nathan Foster, Michael B. Greenwald, Jonathan T. Moore, Benjamin C. Pierce, and Alan Schmitt. 2007. Combinators for Bidirectional Tree Transformations: A Linguistic Approach to the View-Update Problem. ACM Transactions on Programming Languages and Systems 29, 3 (2007), 17. https://doi.org/10.1145/1232420.1232424

Nate Foster, Kazutaka Matsuda, and Janis Voigtländer. 2012. Three Complementary Approaches to Bidirectional Programming In Generic and Indexed Programming. Lecture Notes in Computer Science, Vol. 7470. Springer, 1-46. https://doi.org/10. 1007/978-3-642-32202-0_1

Jeremy Gibbons. 2007. Datatype-Generic Programming. In Datatype-Generic Programming. Lecture Notes in Computer Science, Vol. 4719. Springer, 1-71. https://doi.org/10.1007/978-3-540-76786-2_1

C. A. R. Hoare. 1969. An Axiomatic Basis for Computer Programming. Commun. ACM 12, 10 (1969), 576-580. https: //doi.org/10.1145/363235.363259

Martin Hofmann, Benjamin Pierce, and Daniel Wagner. 2011. Symmetric Lenses. In Symposium on Principles of Programming Languages (POPL'11). ACM, 371-384. https://doi.org/10.1145/1925844.1926428

Zhenjiang Hu and Hsiang-Shang Ko. 2017. Principles and Practice of Bidirectional Programming in BiGUL. Draft lecture notes for the Oxford Summer School on Bidirectional Transformations. https://bitbucket.org/prl_tokyo/bigul/raw/master/ SSBX16/tutorial.pdf

Zhenjiang Hu, Hugo Pacheco, and Sebastian Fischer. 2014. Validity Checking of Putback Transformations in Bidirectional Programming. In International Symposium on Formal Methods (Lecture Notes in Computer Science), Vol. 8442. Springer, 1-15. https://doi.org/10.1007/978-3-319-06410-9_1

Daisuke Kinoshita and Keisuke Nakano. 2017. Bidirectional Certified Programming. In International Workshop on Bidirectional Transformations (BX'17). CEUR-WS.org, 31-38. http://ceur-ws.org/Vol-1827/paper7.pdf

Hsiang-Shang Ko, Tao Zan, and Zhenjiang Hu. 2016. BiGUL: A Formally Verified Core Language for Putback-Based Bidirectional Programming. In Workshop on Partial Evaluation and Program Manipulation (PEPM'16). ACM, 61-72. https://doi.org/10.1145/2847538.2847544

Nuno Macedo, Hugo Pacheco, Alcino Cunha, and José N. Oliveira. 2013. Composing Least-Change Lenses. In International Workshop on Bidirectional Transformations (Electronic Communications of the EASST). EASST. https://doi.org/10.14279/tuj. eceasst.57.868

Kazutaka Matsuda and Meng Wang. 2015. Applicative Bidirectional Programming with Lenses. In International Conference on Functional Programming (ICFP'15). ACM, 62-74. https://doi.org/10.1145/2858949.2784750

James McKinna. 2016. Bidirectional Transformations are Proof-Relevant Bisimulations (Extended Abstract). In International Workshop on Type-Driven Development (TyDe'16). http://groups.inf.ed.ac.uk/bx/TyDe16.pdf 
Jorge Mendes, Hsiang-Shang Ko, and Zhenjiang Hu. 2016. The Under-Appreciated Put: Implementing Delta-Alignment in BiGUL. Technical Report GRACE-TR 2016-03. GRACE Center, National Institute of Informatics. http://grace-center.jp/ wp-content/uploads/2016/04/GRACE-TR-2016-03.pdf

Hugo Pacheco, Alcino Cunha, and Zhenjiang Hu. 2012. Delta Lenses over Inductive Types. In International Workshop on Bidirectional Transformations. EASST. https://doi.org/10.14279/tuj.eceasst.49.713

Hugo Pacheco, Zhenjiang Hu, and Sebastian Fischer. 2014a. Monadic Combinators for "Putback" Style Bidirectional Programming. In Workshop on Partial Evaluation and Program Manipulation (PEPM'14). ACM, 39-50. https://doi.org/10. 1145/2543728.2543737

Hugo Pacheco, Tao Zan, and Zhenjiang Hu. 2014b. BiFluX: A Bidirectional Functional Update Language for XML. In International Symposium on Principles and Practice of Declarative Programming (PPDP'14). ACM, 147-158. https://doi.org/ $10.1145 / 2643135.2643141$

John C. Reynolds. 2002. Separation Logic: A Logic for Shared Mutable Data Structures. In Symposium on Logic in Computer Science (LICS'02). IEEE, 55-74. https://doi.org/10.1109/LICS.2002.1029817

Perdita Stevens. 2010. Bidirectional Model Transformations in QVT: Semantic Issues and Open Questions. Software and Systems Modeling 9 (2010), 7. https://doi.org/10.1007/s10270-008-0109-9

Michael Kirkedal Thomsen and Holger Bock Axelsen. 2015. Interpretation and Programming of the Reversible Functional Language RFUN. In Symposium on the Implementation and Application of Functional Programming Languages (IFL'15). ACM. https://doi.org/10.1145/2897336.2897345

Niki Vazou, Eric L. Seidel, Ranjit Jhala, Dimitrios Vytiniotis, and Simon Peyton Jones. 2014. Refinement Types for Haskell. In International Conference on Functional Programming (ICFP'14). ACM, 269-282. https://doi.org/10.1145/2628136.2628161

Janis Voigtländer, Zhenjiang Hu, Kazutaka Matsuda, and Meng Wang. 2013. Enhancing Semantic Bidirectionalization via Shape Bidirectionalizer Plug-ins. Journal of Functional Programming 23, 5 (2013), 515-551. https://doi.org/10.1017/ S0956796813000130

Tao Zan, Li Liu, Hsiang-Shang Ko, and Zhenjiang Hu. 2016. BRuL: A Putback-Based Bidirectional Transformation Library for Updatable Views. In International Workshop on Bidirectional Transformations (BX'16). CEUR-WS.org, 77-89. http: //ceur-ws.org/Vol-1571/paper_3.pdf

Weize Zhao, Haiyan Zhao, and Zhenjiang Hu. 2016. A Framework for Synchronization between Feature Configurations and Use Cases based on Bidirectional Programming. In International Model-Driven Requirements Engineering Workshop (MoDRE'16). IEEE, 170-179. https://doi.org/10.1109/REW.2016.040

Zirun Zhu, Yongzhe Zhang, Hsiang-Shang Ko, Pedro Martins, João Saraiva, and Zhenjiang Hu. 2016. Parsing and Reflective Printing, Bidirectionally. In International Conference on Software Language Engineering (SLE'16). ACM, 2-14. https: //doi.org/10.1145/2997364.2997369 